CERN-TH/95-221

arch-ive/9509171

\title{
SYMMETRY BREAKING AT ENHANCED SYMMETRY POINTS
}

\author{
Michael B. Green and Michael Gutperle \\ Theory Division, CERN, \\ CH-1211, Geneva 23, Switzerland \\ and \\ DAMTP, Silver Street, \\ Cambridge CB3 9EW, UK
}

\begin{abstract}
The influence of world-sheet boundary condensates on the toroidal compactification of bosonic string theories is considered. At the special points in the moduli space at which the closed-string theory possesses an enhanced unbroken $G \times G$ symmetry (where $G$ is a semi-simple product of simply laced groups) a scalar boundary condensate parameterizes the coset $G \times G / G$. Fluctuations around this background define an open-string generalization of the corresponding chiral nonlinear sigma model. Tree-level scattering amplitudes of on-shell massless states ('pions') reduce to the amplitudes of the principal chiral model for the group $G$ in the low energy limit. Furthermore, the condition for the vanishing of the renormalization group beta function at one loop results in the familiar equation of motion for that model. The quantum corrections to the open-string theory generate a mixing of open and closed strings so that the coset-space pions mix with the closed-string $G \times G$ gauge fields, resulting in a Higgs-like breakdown of the symmetry to the diagonal $G$ group. The case of non-oriented strings is also discussed.
\end{abstract}

CERN-TH/95-221

August 1995

\footnotetext{
${ }^{0}$ M.B.Green@damtp.cam.ac.uk $\quad$ M.Gutperle@damtp.cam.ac.uk
} 


\section{Introduction}

The study of conformal field theory on two-dimensional manifolds with boundaries has several interesting applications. The one that has most relevance to this paper is the description of the perturbation theory of interacting open and closed bosonic strings, in which the two-dimensional manifold describes the world-sheet embedded in $D$-dimensional space-time.

We will be concerned with the rôle of extra massless open-string states that arise by compactification of open-string theories. A generic toroidal compactification of closedstring theory on a $d$-dimensional torus possesses $2 d$ massless $U(1)$ gauge potentials, resulting in a $U(1)^{d} \times U(1)^{d}$ gauge symmetry. These gauge fields are excitations around the condensates of background metric and antisymmetric tensor fields, $G_{\mu i}$ and $B_{\mu i}$ (where $i$ labels the compactified directions and $\mu$ labels the space-time dimensions). There are also $d^{2}$ massless scalar closed-string states associated with the $O(d, d) /(O(d) \times O(d))$ moduli space of toroidal compactifications. In addition, a theory with open strings has $d$ extra massless scalar states arising from compactification of the massless open-string vector state - the excitation of the boundary condensate of the background open-string vector potential.

The symmetry of the closed-string theory is well-known to be enhanced on special sub-manifolds of the background moduli space so it is of interest to study the spectrum of the open-string sector in the same backgrounds in a theory with world-sheet boundaries. For the purposes of this paper we shall consider the special isolated enhanced symmetry points at which the symmetry is 'maximal' which means that it has the form $G \times G$, where $G$ is a semi-simple product of simply-laced groups $(A, D, E)$ of rank $d$, although many considerations also generalize to non-maximally enhanced symmetry points.

The spectrum of the open-string sector of a conformal field theory is related to the spectrum of the closed-string sector by the modular properties of the theory [1]. Thus, the partition function for a theory defined on an annular world-sheet with boundary conditions $A$ at $\sigma=0$ and $B$ at $\sigma=\pi$ can be expressed as the trace over open-string states,

$$
Z_{A B}(w)=\operatorname{tr}\left(w^{H_{A B}}\right)=\sum_{i} N_{A B}^{i} \chi_{i}(w)
$$

(where $H_{A B}$ is the open-string hamiltonian and $w=e^{2 i \pi \tau^{\prime}}$, where $2 \pi \tau^{\prime}$ is the imaginary proper time around the annulus). The $\chi_{i}(w)$ are the conformal characters of the representation labelled by $i$ of the Virasoro algebra and $N_{A B}^{i}$ are integers counting how often one representation appears in the partition function. A modular transformation $\tau^{\prime} \rightarrow \tau=-1 / \tau^{\prime}$ maps the annulus to a cylinder and the partition function can be expressed as a transition matrix element between the two boundary end-states states,

$$
Z(q)=\left\langle A\left|q^{H_{c l}}\right| B\right\rangle
$$


where $q=e^{i \pi \tau}$ and $H_{c l}$ is the closed-string hamiltonian. This demonstrates that the boundary conditions $A, B, .$. are in one to one correspondence to the boundary states $|A\rangle,|B\rangle, \ldots$

This relation between the annulus and cylinder will be used in section 2 to show that the presence of enhanced affine symmetry at special points in the moduli space of toroidal compactifications of closed-string theory implies the presence of extra massless scalar states in the open-string sector. Vertex operators for the extra massless openstring and closed-string states are given in terms of the affine algebra associated with the enhanced symmetry.

Open-string vertex operators arise from fluctuations in background boundary condensates. The study of conformal field theories in the presence of non-trivial boundary condensates (boundary conformal field theory) has been motivated by several physical applications including monopole-catalysed baryon decay [2], [3], the Kondo effect [4], tunneling in quantum wires [5], dissipative quantum mechanics [6], [7] and the fractional quantum Hall effect [ 8$]$. In these applications the boundary condensate is defined by a marginal boundary term added to the bulk action,

$$
S=S_{0}+h_{b} \int_{\partial \Sigma} d \sigma \psi_{b}(\sigma)
$$

where $\psi_{b}$ is a boundary operator (and $\sigma$ is the coordinate tangential to the boundary). If the boundary term is a truly marginal perturbation to the bulk theory (so that $\psi_{b}$ has scaling dimension one) the resulting theory will still be conformally invariant. The moduli space of the theory will generally include parameters that enter into this boundary term. Several different cases have been studied in the literature. One example [9], [10], is that of a single free compactified boson on the half line with an action,

$$
S=\frac{1}{8 \pi} \int d \sigma d \tau\left(\partial_{\alpha} X\right)^{2}-\int d \sigma \frac{1}{2}\left(g e^{\frac{i}{\sqrt{2}} X(0, \sigma)}+\bar{g} e^{\frac{-i}{\sqrt{2}} X(0, \sigma)}\right)
$$

where $g$ is a (complex) constant parameter. Another example is the coupling of a constant antisymmetric tensor field to the boundary in open string theory [11]

$$
S=\frac{1}{8 \pi} \int d \sigma d \tau\left(\partial_{\sigma} X^{\mu}\right)^{2}+\int d \sigma F_{\mu \nu} \partial_{\tau} X^{\mu}(0, \sigma) X^{\nu}(0, \sigma)
$$

which is also an exact deformation of the bulk theory. The case of two free bosons in the presence of both (田) and (5) has been discussed in [12] and the case of $n$ free bosons in [13]. In all cases the exact solution is given by a modified boundary state $|B\rangle_{g}$ which generally depends on the parameters of the perturbation. However, at points in the moduli space of the bulk theory at which there is enhanced symmetry the boundary conformal field theory may become independent of some (or all) of these parameters. This was used as important tool in solving the boundary scattering problem in [10] and [14] where the boundary 
interaction was fermionized and the theory at the self-dual point was mapped into a theory of free fermions with twisted boundary conditions. In all these cases the condensates were taken to be constant, i.e., the parameters are independent of the position on the boundary, $\sigma$. [Alternatively, the bulk degrees of freedom can be integrated out and the boundary conformal field theory is then expressed as a 1-dimensional reparameterization invariant field theory [15] (an approach that makes contact with dissipative quantum mechanics [7] and has also been used to discuss perturbations like (14) and (5) [16]), 17.]

The more general situation in which the boundary term is not marginal is of importance in defining off-shell string field theory in a background independent manner and is the subject of several interesting papers [18, [19], [20].

In section 3 we will argue that, at the enhanced symmetry points of the toroidally compactified theory constant scalar boundary condensates (such as that in (雨) and generalizations) define coset spaces $G \times G / G$ and open-string vertex operators attached to the boundary describe excitations around these backgrounds. The tree-level S-matrix of the open-string sector will be described and the nonlinear symmetries associated with this coset space model (which is equivalent to the principal chiral model for the group $G$ ) will be demonstrated.

The connection between the open-string tree amplitudes and the nonlinear sigma model is made precise in section 4, where the low-energy limit of the string theory is considered. Firstly, the limit of some elementary S-matrix elements is discussed and shown to correspond to low-order terms in the expansion of the S-matrix of the sigma model. The one-loop beta function for the theory with an arbitrary scalar boundary condensate is then considered. The lowest-order condition for conformal invariance (the vanishing of the one-loop beta function) is shown to coincide with the equation of motion for the sigma model. Higher-order corrections correspond to higher derivative nonlinear sigma models (which also couple to the field strength of the massless open-string 'photon').

The interaction between open and closed strings will be considered in section 5 . This interaction arises naturally from string perturbation theory and it results in a significant mixing between the open and closed sectors. In the uncompactified theory this leads to a Higgs-like mechanism in which the antisymmetric tensor potential in the closed-string sector gains a mass by absorbing the neutral massless vector potential (the 'photon') of the open-string sector. This was pointed out a long time ago [21 and was investigated in more detail in [22, 223]. In the generalization to generic $d$-dimensional toroidal compactifications $d$ linear combinations of the $2 d U(1)$ gauge fields gain mass by absorbing the $d$ extra massless open-string scalar states. The generic $U(1)^{d} \times U(1)^{d}$ gauge symmetry is thereby reduced to a diagonal $U(1)^{d}$. This is a stringy generalization of the abelian Higgs effect. We shall demonstrate that the non-abelian generalization of this effect at enhanced symmetry points. The extra massless scalar open-string states in the coset space $G \times G / G$ are eaten by $\operatorname{dim}(G)$ of the generators of the $G \times G$ enhanced symmetry, giving a non-zero mass to half of the gauge bosons and leaving the diagonal $G$ symmetry unbroken. This 
should be contrasted with the effect in purely closed-string theories where the enhanced gauge symmetries are broken by marginal perturbations away from the enhanced symmetry point, in which case a subset of the gauge particles become massive by mixing with the massless closed-string moduli fields.

The generalization to theories with non-orientable world-sheets is considered in section 6 . In this case the closed-string sector only has the diagonal $G$ symmetry to begin with. At enhanced symmetry points there are two distinct definitions of the projection onto the non-orientable open-string sector. In one of these (which is the same projection as the one used at generic points in moduli space) the massless open-string scalars lie in the coset the coset $G / U(1)^{d}$ (where $d$ is the rank of $G$ ). There is no mixing between the massless states of the open and closed sectors so the diagonal $G$ symmetry again remains as the unbroken gauge symmetry. At the enhanced symmetry points a generalization of this projection is more natural in the fermionic formulation of the theory, which eliminates all the massless open-string scalar states, again leaving an unbroken diagonal $G$ gauge symmetry.

\section{Enhanced symmetry points}

A striking feature of toroidal compactifications of closed-string theory is the occurrence of enhanced gauge symmetry for special values of the moduli of the target-space torus. This section will highlight a corresponding enhancement of symmetry at the same values of the moduli in the open-string sector of the theory in which there are both open and closed strings.

\subsection{Review of closed-string sector}

The $U(1)^{d} \times U(1)^{d}$ world-sheet affine algebra associated with generic compactifications of $d$ free bosons gives rise, in closed-string theory, to target-space gauge fields which are the Kaluza-Klein modes of the antisymmetric tensor and metric fields. The $d$-dimensional toroidal compactification of the target space on the lattice $\Lambda=2 \pi Z^{d}$ is encoded in the background fields $G, B$ which parameterize the moduli space of toroidal compactifications $O(d, d ; R) /(O(d, R) \times O(d, R))$ [25]. Each modulus is a massless scalar closed-string state so the generic number of massless scalars in the closed-string sector is the dimensionality of the moduli space, $d^{2}$. The action containing the constant background fields is given by,

$$
S_{d}=\frac{1}{4 \pi \alpha^{\prime}} \int d^{2} z \partial_{\alpha} X^{i} \partial_{\beta} X^{j}\left(\eta^{\alpha \beta} G_{i j}+\epsilon^{\alpha \beta} B_{i j}\right)
$$

(reparametrization ghosts are incorporated in the standard manner and will not be discussed explicitly here). The term in the action containing the $B$ field is a total world-sheet 
derivative and shifts the left and right moving momenta $p, \bar{p}$ in the compactified dimensions,

$$
p_{i}=\left(\Pi_{i}+(G-B)_{i j} L^{j}\right) \quad \bar{p}_{i}=\left(\Pi_{i}-(G+B)_{i j} L^{j}\right) .
$$

Here $L^{i}$ is in the lattice $\Lambda$ while $\Pi_{i}$ denotes the canonical momentum which takes values on the dual lattice, $\Lambda^{*}=Z^{d}$ (and the choice $\alpha^{\prime}=1$ has been made for convenience). The physical state conditions are,

$$
\left(L_{0}-1\right)|\Phi\rangle=0=\left(\tilde{L}_{0}-1\right)|\Phi\rangle,
$$

where the zero Virasoro modes are defined by,

$$
L_{0}=\frac{1}{4} k^{2}+\frac{1}{4} p^{2}+N, \quad \tilde{L}_{0}=\frac{1}{4} k^{2}+\frac{1}{4} \bar{p}^{2}+\tilde{N},
$$

( $k^{\mu}$ denotes the uncompactified momentum in the $26-d$ dimensions and $N, \tilde{N}$ are the level numbers in the left-moving and right-moving sectors). The closed string hamiltonian $L_{0}+\tilde{L}_{0}$ is given by inserting (7) into (9),

$$
L_{0}+\tilde{L}_{0}=\frac{1}{2} k^{2}+N+\tilde{N}+\frac{1}{2} \prod_{i} G^{i j} \Pi_{j}+\frac{1}{2} L^{i}\left(G-B G^{-1} B\right)_{i j} L^{j}+L^{i} B_{i j} G^{j k} \Pi_{k} .
$$

The ocurrence of extra massless vector states at special values of the internal momenta signals the enhanced gauge symmetry, which is associated with an enhanced worldsheet affine algebra. If one dimension is compactified to the self-dual radius that is the fixed point of the T-duality transformation, $R \rightarrow 1 / R$, the enhanced symmetry is $S U(2) \times S U(2)$. Higher-dimensional toroidal compactifications are best described in terms of constant non-trivial background fields, $G_{i j}$ and $B_{i j}$, and the maximally enhanced symmetry points are generalized fixed points of the $T$-duality group, $O(d, d ; Z)$. If $B=0$ the enhanced symmetry $S U(2)^{d} \times S U(2)^{d}$ arises but more generally semi-simple products of simply-laced Lie algebras (i.e., of type $A, D, E)$ of total rank $d$ can be obtained. The maximal symmetry $G \times G$ (where $G$ is a simply-laced Lie algebra of rank $d$ ) is achieved by choosing the background fields in the following way [26]. Let $C_{i j}$ denote the Cartan matrix of the simply-laced Lie algebra $G$ and define the background fields as $G_{i j}=1 / 2 C_{i j}$ and $B_{i j}=G_{i j}$ for $i>j, B_{i j}=-G_{i j}$ for $i<j$ and $B_{i i}=0$. With these choices $E \equiv G+B \in S L(d, Z)$. The points in the compactification moduli space with maximal enhanced gauge symmetry are generalized fixed points under the duality transformation $O(d, d ; Z)$ generated by a combination of $S L(d, Z)$ conjugation by a matrix $M$ and a shift of $B_{i j}$ by an antisymmetric integer-valued matrix, $\Theta$. This transforms $E$ to $E^{\prime}=M^{t}(E+\Theta) M$. For example, in the special case with $M=E^{-1}$ and $\theta=E^{t}-E$, duality reduces to $E \rightarrow E^{\prime}=E^{-1}$. The extra gauge potentials that arise at points of enhanced symmetry are labelled by roots in the Lie algebra lattice of $G$. 
The condition that there is an enhancement of the gauge symmetry (i.e., extra massless closed-string vector states) requires either $p=0, \bar{p}^{2}=4, N=1$ and $\tilde{N}=0$ or $\bar{p}=0$, $p^{2}=4, N=0$ and $\tilde{N}=1$. In either case it follows from (9) and (7) that the condition for enhanced symmetry in the closed-string sector is

$$
L^{i} G_{i j} L^{j}=1 .
$$

In addition to the enhancement of the number of massless gauge particles there are $(\operatorname{dim} G)^{2}$ extra massless closed-string scalar states at a maximally enhanced symmetry point with symmetry $G \times G$. As is well understood, if the theory is deformed away from such an enhanced symmetry point $4 d^{2}-4 d$ of these scalars are eaten by the extra massless vectors which then become massive and the rest of the scalars also gain mass apart from the $d^{2}$ that remain massless at generic compactifications. In other words, the enhanced symmetry is spontaneously broken by deformations away from the enhanced symmetry point. The main focus in this paper is on a mechanism that operates in the presence of boundaries that breaks the enhanced symmetry without this deformation.

\subsection{Boundary states in the absence of a boundary condensate}

Since the constant $B_{i j}$ term in the action is a total derivative it gives a boundary term in theories with world-sheet boundaries,

$$
S_{B}=\frac{1}{4 \pi} \int_{\Sigma} d^{2} \sigma \epsilon^{\alpha \beta} \partial_{\alpha} X^{i} \partial_{\beta} X^{j} B_{i j}=\frac{1}{4 \pi} \oint_{\partial \Sigma} d \sigma \partial_{\sigma} X^{i} B_{i j} X^{j},
$$

where $\partial_{\sigma}$ denotes the tangential derivative on the boundary. The term (12) is equivalent to coupling a constant background magnetic field to the boundaries of a neutral open string (a string with charges of equal magnitude and opposite sign at each end). The string coordinate $X^{i}$ now satisfies the free boundary conditions, $\left.\left(G_{i j} \partial_{\tau} X^{j}+B_{i j} \partial_{\sigma} X^{j}\right)\right|_{\partial_{\Sigma}}=0$, which guarantee the conservation of the world-sheet energy-momentum $(T(z)=\bar{T}(\bar{z})$ when the world-sheet is the upper-half plane). If the world-sheet with a boundary is parameterized as a semi-infinite cylinder these boundary conditions are operator conditions on the boundary state at the end of the cylinder, i.e.,

$$
\left(G_{i j} \partial_{\tau} X^{i}+B_{i j} \partial_{\sigma} X^{j}\right)|B\rangle=0,
$$

and the modes of the energy-momentum tensor satisfy

$$
\left(L_{n}-\tilde{L}_{-n}\right)|B\rangle=0,
$$

where $L_{n}$ and $\tilde{L}_{n}$ are the Virasoro modes. 
The boundary state in the cylinder frame that satisfies (13) can be constructed in terms of the modes of the closed-string coordinates, $\alpha_{n}^{i}$ and $\tilde{\alpha}_{n}^{i}$. The state is given by [27], [28], [1],

$$
|B\rangle=C \exp \left(-\sum_{n>0} \frac{1}{n} \alpha_{-n}^{i} M_{i j} \tilde{\alpha}_{-n}^{j}\right) \sum_{L \in Z^{d}}\left|p_{i}, \bar{p}_{i}\right\rangle
$$

(again ignoring the terms containing the reparametrization ghosts) where,

$$
p_{i}=(G-B)_{i j} L^{j}, \quad \bar{p}_{i}=-(G+B)_{i j} L^{j}
$$

and

$$
M^{i}{ }_{j}=\left[(G+B)(G-B)^{-1}\right]_{j}^{i}
$$

is an element of $S O(d, R)$. The effect of the boundary term is a rotation in the compactified space of the left-movers with respect to the right-movers,

$$
\left(X^{i}(\sigma)-M_{j}^{i} \bar{X}^{j}(-\sigma)\right)|B\rangle=0
$$

The sum over the lattice vectors ensures that the total canonical momentum entering or leaving the boundary vanishes, i.e. $\Pi_{i}=0$. The normalization constant $C$ of the boundary state can be determined by exploiting the modular properties of the one-loop open-string partition function as will be seen below.

In the case of non-orientable open-string theories a general world-sheet is a Riemann surface with an arbitrary number of cross-caps in addition to boundaries and handles. A cross-cap can be represented by a state, $|C\rangle$, at the end of a cylinder on which the coordinates satisfy $(X(\sigma)-X(\pi+\sigma))|C\rangle=0$ and $\left(\partial_{\tau} X(\sigma)+\partial_{\tau} X(\pi+\sigma)\right)|C\rangle=0$.

\subsection{Massless open-string states and modular transformations}

We shall now turn to consider the spectrum of open strings. The internal non-abelian symmetry will come from compactification rather than from Chan-Paton factors at the string end-points and since we are dealing with the bosonic theory the spectrum will inevitably contain a tachyon and a massless gauge particle - a 'photon'. For most of the paper we shall be considering the orientable open-string theory in which non-orientable world-sheets (such as Möbius strips and Klein bottles) are eliminated by including a rather trivial $U(1)$ Chan-Paton factor. Although the photon is present in this case all states are neutral under its $U(1)$ and it will decouple in the low energy limit. In the absence of any Chan-Paton factors the theory is non-orientable and the photon is absent.

At particular points in moduli space, the space of $B$ and $G$, extra massless openstring scalar states arise at precisely the same points as the enhanced symmetry points of the closed-string sector. This can be seen in a particularly direct manner by considering modular transformations of the annulus diagram. On the one hand this diagram may 
be viewed as a loop of open string while it may also be viewed as a cylindrical worldsheet which describes a closed string propagating between an initial and a final boundary state. As described in the introduction these two descriptions are related by a modular transformation that thereby relates the spectrum of the open-string sector to that of the closed string.

The annulus diagram (the one-loop partition function for an open string) with background $B$ and $G$ fields and is given by the trace over open-string states,

$$
Z(B, G)=\int \frac{d t}{t} Z_{o p}(w)=\int \frac{d t^{\prime}}{t^{\prime}} \operatorname{tr}\left(w^{L_{o}^{o p}-1}\right)=\int \frac{d t^{\prime}}{t^{\prime}} \int d^{D} p \sum_{k=0}^{\infty} N_{k} w^{k+p^{2}-1}
$$

where $D=26-d, L_{0}^{\text {open }}$ is the open-string hamiltonian, $t^{\prime}=-i \tau^{\prime}$ is the proper (euclidean) time in the open-string channel and $w=e^{2 i \pi t^{\prime}} . N_{k}$ is the number of open-string states with mass $m^{2}=k-1$. This function $Z$ can also be calculated by expressing the partition function as the matrix element of a closed string propagating between two boundary states,

$$
\begin{aligned}
Z(B, G) & =\int d t Z_{c l}(q)=\int d t\left\langle B\left|q^{L_{0}+\tilde{L}_{0}-2}\right| B\right\rangle \\
& =C^{2} \int d t q^{-2} \prod_{n=1}^{\infty}\left(1-q^{2 n}\right)^{-24} \sum_{m \in Z^{d}} q^{\frac{1}{2} m^{i}\left(G-B G^{-1} B\right)_{i j} m^{j}}
\end{aligned}
$$

where $t=-i \tau$ is the proper (euclidean) time in the cylinder channel and $q=\exp (i \pi t)$. The equality of the two expressions for $Z$ is made manifest by the change of variables in (20) from $\tau$ to $\tau^{\prime}=-1 / \tau$ which maps the cylinder into the annulus. After this change of variables and a Poisson resummation of the sum over the winding modes (20) becomes,

$$
\begin{aligned}
& Z(B, G)=\int d \tau^{\prime} Z_{c l}^{\prime}(w) \\
& =C^{2} \int \frac{d \tau^{\prime} 2^{d / 2}}{\left(i \tau^{\prime}\right)^{14-\frac{d}{2}}} \operatorname{det}\left(G-B G^{-1} B\right)^{-\frac{1}{2}} w^{-1} \prod_{n=1}^{\infty}\left(1-w^{n}\right)^{-24} \sum_{n \in Z^{d}} w^{n^{i}\left(G-B G^{-1} B\right)_{i j}^{-1} n^{j}}(21)
\end{aligned}
$$

Comparison of (19) and (21) determines the mass spectrum of the open-string states in terms of the bulk background fields, $G$ and $B$, of the closed-string sector. Explicitly, the spectrum of massless open-string states is given by the $w^{0}$ terms in (20). The expansion of $\left(1-w^{n}\right)^{-24}$ leads to the usual states of the massless photon and the $d$ scalars that come from its compactified components. Additionally, the condition that there is an enhancement in the spectrum of massless scalar open strings is seen from the last factor to be,

$$
n_{i}\left[\left(G-B G^{-1} B\right)^{-1}\right]^{i j} n_{j}=1 .
$$


This should be compared with the condition (11) for an enhancement in the closed-string massless spectrum. Using $\left(G-B G^{-1} B\right)^{-1}=\left(E^{-1}\right)^{t} G E^{-1}$, where $E=G+B$ it is evident that the solutions of equations (22) and (11) are proportional,

$$
n_{i}=(G+B)_{i j} L^{j} .
$$

This shows that the condition on $B$ and $G$ for an enhancement of the spectrum of massless open-string scalar states is precisely the same as the condition for the enhancement of the spectrum of closed-string massless vector states. This argument is valid whenever the symmetry is enhanced (not just at the self-dual points of maximal symmetry).

The open-string spectrum can also be determined directly as the spectrum of the open-string hamiltonian,

$$
L_{0}^{\text {open }}=\Pi_{i}\left[\left(G-B G^{-1} B\right)^{-1}\right]^{i j} \Pi_{j}+k^{2}+N,
$$

where the canonical momentum, $\Pi_{i} \in Z^{d}$, again demonstrating the extra open-string states satisfying $L_{0}^{\text {open }}$ when (22) is satisfied.

\subsection{Vertex operators for the massless states}

(a) The bosonic construction

The level-one Kac-Moody algebra can be represented in terms of bosons by the FrenkelKac-Segal construction of the group $G_{L} \times G_{R}$. The left-moving currents are given (in the cylinder channel with $\left.z=e^{i(\tau+\sigma)}\right)$ by,

$$
H^{i}(z)=\partial X^{i}, \quad E^{\lambda}(z)=c(\lambda): e^{i \lambda^{i} X^{i}(z)}:
$$

while the right-moving currents are,

$$
\tilde{H}^{i}(\bar{z})=M^{i}{ }_{j} \bar{\partial} X^{j}, \quad \tilde{E}^{\lambda}(\bar{z})=-\tilde{c}(\lambda): e^{i \lambda^{j} M_{i j} \bar{X}^{i}(\bar{z})}: .
$$

Here $H^{i}, \tilde{H}^{i}$ denote the currents in the Cartan subalgebra of $G_{L}$ and $G_{R}$ respectively. The $E^{\lambda}$ are constructed with winding number states and $\lambda$ are roots of the Lie algebra of $G$. The cocycles $c(\lambda)$ are constructed in terms of the zero modes of $X$ and $\bar{X}$. The root lattice for the right-moving algebra has been rotated with the orthogonal matrix $M^{i}{ }_{j}$, which leaves the operator product expansions unaltered. The currents, $J_{L}^{a}(\sigma)$, that satisfy the algebra,

$$
\left[J_{L}^{a}(\sigma), J_{L}^{b}\left(\sigma^{\prime}\right)\right]=f^{a b c} J_{L}^{c}(\sigma) \delta\left(\sigma-\sigma^{\prime}\right)+\delta^{a b} \delta^{\prime}\left(\sigma-\sigma^{\prime}\right)
$$

are linear combinations of the left-moving currents $H, E$, where $f^{a b c}$ are the structure constants for the Lie algebra $G_{L}$. The same construction also applies to the right-moving currents that are functions of $\bar{z}$. 
In situations in which there are affine symmetries the left-moving and right-moving currents the boundary conditions on the bosonic fields translate into conditions on the currents, $J_{L}^{a}(z)-J_{R}^{a}(\bar{z})=0$, at $z=\bar{z}$ on the half-plane. Transforming to the semi-infinite cylinder this translates into the condition on the boundary state,

$$
\left(J_{L n}^{a}+J_{R-n}^{a}\right)|B\rangle=0
$$

(where the modes are defined by, $J^{a}(\sigma)=\sum_{n} J_{n}^{a} e^{i n \sigma}$ ). The plus sign in this equation arises from the rotation of the weight-one currents through $\pi / 2$ in transforming from the half plane to the cylinder frame.

The vertex operators for the massless closed-string vector states are given by,

$$
V_{k}^{a, \mu}(z)=J_{R}^{a}(\bar{z}) \partial X^{\mu}(z) e^{i k \cdot(X(z)+\bar{X}(\bar{z}))}, \quad \tilde{V}_{k}^{a, \mu}(z)=J_{L}^{a}(z) \bar{\partial} \bar{X}^{\mu}(\bar{z}) e^{i k \cdot(X(z)+\bar{X}(\bar{z}))} .
$$

The vertex operators for the massless open-string scalar states are attached to the boundary (at, say, $\tau=0$ ). They can be written in the closed-string channel as,

$$
S_{k}^{a}(\sigma)=J_{L}^{a}(\sigma) e^{i k \cdot X(\sigma)} \equiv \frac{1}{2}\left(J_{L}^{a}(\sigma)-J_{R}^{a}(\sigma)\right) e^{i k \cdot X(\sigma)},
$$

where the second expression follows from the fact that the open-string vertices are attached to the boundary and using $\left(J_{L}^{a}(\sigma)+J_{R}^{a}(\sigma)\right)|B\rangle=0$. The massless vector vertex is given by,

$$
V_{k}^{\mu}(\sigma)=\partial_{\sigma} X^{\mu}(\sigma) e^{i k \cdot X(\sigma)}
$$

(where $\sigma$ is again the parameter tangential to the boundary).

(b) The fermionic construction

Level-one affine algebras also have well-known representations in terms of free fermions [29]. For simplicity we will here consider the special case of $S O(2 d) \times S O(2 d)$ which is implemented by $2 d$ left-moving and $2 d$ right-moving Majorana fermions, $\psi_{L}^{i}$ and $\psi_{\underline{R}}^{i}(i=$ $1, \cdots, 2 d)$. These fermions are described by the bulk world-sheet action, $\int d^{2} z\left(\psi_{L}^{i} \bar{\partial} \psi_{L}^{i}+\right.$ $\left.\psi_{R}^{i} \partial \psi_{R}^{i}\right)$ and the currents satisfying (27) can be written as bilinears in real left-moving and right-moving fermions, $\psi_{L}^{i}, \psi_{R}^{i}\{i=1, . ., 2 d\}$,

$$
J_{L}^{a}(z)=\psi_{L}^{i}(z) T_{i j}^{a} \psi_{L}^{j}(z), \quad J_{R}^{a}(z)=\psi_{R}^{i}(z) T_{i j}^{a} \psi_{R}^{j}(z)
$$

where $T_{i j}^{a}$ are the antisymmetric generators of $S O(2 d)$. Quantizing in the cylinder channel the massless states of the adjoint representation lie in the sector with antiperiodic boundary conditions on both fermions (the NS/NS sector). The closed-string boundary state in this sector is given by,

$$
|B\rangle=P_{G S O} \exp \left(i \sum_{n \in Z+1 / 2} \psi_{L-n}^{i} \psi_{R-n}^{i}\right)|B\rangle_{0}
$$


(where $|B\rangle_{0}$ is the boundary state involving the space-time bosons). The GSO projector is given by $\left(1+(-1)^{F}\right)\left(1+(-1)^{\tilde{F}}\right) / 4$, where $F$ and $\tilde{F}$ are the fermion number operators in the left-moving and right-moving sectors. This projection eliminates states with odd numbers of $\psi$ or $\tilde{\psi}$ excitations, resulting in a theory that is equivalent to that given by the bosonic description. The state (33) implements the boundary condition,

$$
\left(\psi_{L n}^{i} \pm i \psi_{R-n}^{i}\right)|B\rangle=0
$$

which is the boundary condition on the closed-string states that corresponds to the condition at an end-point of an open string, $\psi_{L}^{i}= \pm \psi_{R}^{i}$. The relative factor of $i$ appears in (33) from the conformal transformation that rotates the coordinate frame through $\pi / 2$ in transforming conformal spin-1/2 fields from the open-string to the closed-string frame. The boundary condition on the currents, (28) also follows from this definition of the boundary state.

The different fermionic spin structures give rise to different $S O(2 d)$ conjugacy classes of $S O(2 d)$. The complete set of states includes the $G S O$-projected $R / R$ and $N / R$ sectors which lead to spinor and bi-spinor representations (which do not have massless excitations).

The vertex operators of (29)-(31) have obvious descriptions in terms of the fermion representation of the currents.

\section{Boundary condensates}

\subsection{Constant condensates}

The occurrence of extra massless open-string states at the points in moduli space at which the gauge symmetry of the closed-string sector is enhanced indicates the presence of new marginal boundary operators. Such operators come from states located on the boundary associated with terms in the action of the form (in the bosonic formalism),

$$
S_{B}=\left.\int d \sigma\left(\sum_{\lambda} g_{\lambda} e^{i \lambda_{i} X^{i}}+\sum_{i} g_{i} \partial_{\sigma} X^{i}\right)\right|_{\tau=0}
$$

(where $g_{\lambda}, g_{i}$ are constants), which gives rise to the enhanced symmetry when $\lambda_{i}$ takes special values. In [13 it was shown these values arise when $\hat{\lambda}_{i}$ are roots of the Lie algebra

$G$, where $\hat{\lambda}_{i}=\left(\delta_{j}^{i}+M^{i}{ }_{j}\right) \lambda^{j}$ (and $M^{i}{ }_{j}$ was defined in (17)) This leads to a modified boundary state in the cylinder channel, $|B\rangle_{g}=\exp \left(S_{B}\right)|B\rangle$ as can be seen by turning the $X^{i}$ modes into left-movers using the boundary condition (18). The effect of the boundary state is then simply a rotation with respect to the left-moving zero modes of the currents,

$$
|B\rangle_{g}=\exp \left(g_{\hat{\lambda}} E_{0}^{\hat{\lambda}}+g_{j} H_{0}^{j}\right)|B\rangle
$$


In terms of the currents $J_{L}^{a}$ this condition can be written as

$$
|B\rangle_{g}=\exp \sum_{a} g_{a} J_{L 0}^{a}|B\rangle .
$$

It follows that a non-zero condensate changes the boundary condition (28) into,

$$
\left(e^{g_{a} J_{L 0}^{a}} J_{L n}^{a} e^{-g_{a} J_{L 0}^{a}}+J_{R-n}^{a}\right)|B\rangle_{g}=0 .
$$

In the fermionic formulation of the current algebra the action of boundary condensate (37) is described by a change in the boundary conditions on the fermions,

$$
\left(\left(e^{-g_{a} T^{a}}\right)_{i j} \psi_{L n}^{j}+i \psi_{R-n}^{i}\right)|B\rangle_{g} .
$$

These boundary conditions also follow directly from the fermionic version of the boundary action on the half plane, $z=\tau+i \sigma(\sigma \geq 0)$,

$$
S_{B}=\int d \sigma \sum_{a} \frac{g_{a}}{2}\left(\psi_{L}^{i} T_{i j}^{a} \psi_{L}^{j}+\psi_{R}^{i} T_{i j}^{a} \psi_{R}^{j}\right)
$$

as the following argument shows. Together with the bulk action this may be written in terms of left-moving fermions only on the whole complex plane by making the standard identification, $\psi_{R}(z)=\psi_{L}(\bar{z})$. The boundary is thereby replaced by an interaction located at $\sigma=0$. The equation of motion for the left-moving $\psi_{L}^{i}$ is then given by,

$$
\left(\partial_{\tau}-\partial_{\sigma}\right) \psi_{L}^{i}+g_{a} T_{i j}^{a} \psi_{L}^{j} \delta(\sigma)=0 .
$$

The delta function may be regularized by replacing it with $1 / 2 a[\Theta(\sigma-a) \Theta(a-\sigma)]$, which is nonzero in the small interval $\sigma \in[-a,+a]$ with $a \rightarrow 0$ so that for $\sigma<a$ and $\sigma>a, \psi_{L}$ satisfies the free equation of motion. In the interval $\sigma \in[-a,+a]$ the perturbation is just a constant matrix. Fourier transforming $\psi_{L}(\tau, \sigma)$,

$$
\psi_{L}^{i}(\sigma, \tau)=\int d \nu \psi_{L \nu}^{i}(\sigma) e^{-i \nu \tau}
$$

leads to a differential equation,

$$
\frac{d}{d \sigma} \psi_{L \nu}^{i}(\sigma)=\left(-i \nu \delta_{i j}+\frac{g_{b}}{2 a} T_{i j}^{b}\right) \psi_{L \nu}^{j}(\sigma)
$$

in the interval $\sigma \in[-a,+a]$. Integrating this between $\sigma=-a$ and $\sigma=a$ gives

$$
\psi_{L}^{i}(a)=\exp (i \nu a+g T)_{i j} \psi_{L}^{j}(-a) .
$$

In the limit $a \rightarrow 0$,

$$
\psi_{L}^{i}\left(0^{+}, \tau\right)=\left(e^{g_{a} T^{a}}\right)_{i j} \psi_{L}^{j}\left(0^{-}, \tau\right)=\left(e^{g_{a} T^{a}}\right)_{i j} \psi_{R}^{j}\left(0^{+}, \tau\right),
$$

where $\sigma=0^{ \pm}$indicates that the line $\sigma=0$ is approached from positive and negative values, respectively. This leads to the relation between left-moving and right-moving fermions in (39) (the factor of $i$ was explained earlier) so that the boundary interaction can be expressed as a change of the fermionic boundary condition. 


\subsection{Coset space interpretation of boundary condensate}

As can be seen from (28) or (38) (for the cases of zero and nonzero boundary condensates respectively) only a particular linear combination of the world-sheet currents is conserved by the boundary. The combinations of the boundary values of the left-moving and rightmoving world-sheet currents ,

$$
\begin{aligned}
& K^{a}(\sigma)=\frac{1}{2}\left(J_{L}^{a}(\sigma)-J_{R}^{a}(\sigma)\right), \\
& J^{a}(\sigma)=\frac{1}{2}\left(J_{L}^{a}(\sigma)+J_{R}^{a}(\sigma)\right),
\end{aligned}
$$

satisfy the commutation relations,

$$
\begin{aligned}
{\left[J^{a}(\sigma), J^{b}\left(\sigma^{\prime}\right)\right] } & =f^{a b c} J^{c}(\sigma) \delta\left(\sigma-\sigma^{\prime}\right)+\delta^{a b} \delta^{\prime}\left(\sigma-\sigma^{\prime}\right), \\
{\left[J^{a}(\sigma), K^{b}\left(\sigma^{\prime}\right)\right] } & =f^{a b c} K^{c}(\sigma) \delta\left(\sigma-\sigma^{\prime}\right), \\
{\left[K^{a}(\sigma), K^{b}\left(\sigma^{\prime}\right)\right] } & =f^{a b c} J^{c}(\sigma) \delta\left(\sigma-\sigma^{\prime}\right)+\delta^{a b} \delta^{\prime}\left(\sigma-\sigma^{\prime}\right) .
\end{aligned}
$$

It follows from (28) that $J$ vanishes on the boundary state while $K$ generates an infinitesimal shift of the boundary condensate. Therefore these transformations define a symmetric space, where $J$ parameterizes the unbroken group $\mathcal{H}$ while $K$ parameterizes the coset $\mathcal{G} / \mathcal{H}$. In the case of interest here $\mathcal{G}=G_{L} \times G_{R}$ and $\mathcal{H}=G_{\text {diagonal }}$. The manifold of possible boundary states (parameterized by the couplings $g_{a}$ ) define equivalent vacua of the theory, transformed into each other by $K$. The massless scalar open-string states are fluctuations around a given vacuum and are the Goldstone bosons of the spontaneously broken symmetry. We will see later that the presence of a boundary causes a breaking of the local $G \times G$ symmetry of the closed-string sector of the theory.

\subsection{The S-matrix of the Goldstone bosons}

The Goldstone bosons describing the fluctuations around a given vacuum state (a state with a given constant condensate) are the massless scalar states of the open string sector described by the scalar vertex operators, $S_{k}^{a}$, (30). Open-string tree amplitudes are obtained by functional integration on a world-sheet that has the topology of a disk, while higher-order diagrams have extra boundaries as well as handles. The on-shell amplitude for $n$ massless scalar open-string states with momenta $k_{i}$ satisfying $k_{i}^{2}=0$ is given by,

$$
A_{n}\left(\Phi_{1}\left(k_{1}\right), \cdots, \Phi_{n}\left(k_{n}\right)\right)=\Phi_{1}^{a_{1}} \cdots \Phi_{n}^{a_{n}}\left\langle a_{1}, k_{1}\left|S_{k_{2}}^{a_{2}} \Delta \cdots \Delta S_{k_{n-1}}^{a_{n-1}}\right| a_{n}, k_{n}\right\rangle+\text { perms. }
$$

where $\Delta$ denotes the open string propagator $\left(L_{0}-1\right)^{-1}$ and $\Phi_{r}^{a_{r}}$ are the momentum-space wave functions of the massless scalar states. Each vertex operator, $S_{k}^{a}$, is proportional to the current $\mathrm{J}^{a}$ at one endpoint or the other of the open string $(\sigma=0, \pi)$. The vertex operator in (30) was expressed in the cylinder channel while those in (49) are in the openstring frame, which leads to a sign change in the combination of currents in the second 
expression in (30), which becomes $\mathrm{J}^{a}(\sigma)=\frac{1}{2}\left(J_{L}^{a}(\sigma)+J_{R}^{a}(\sigma)\right)$. There are no Chan-Paton factors so the expression includes a sum over all non-cyclic permutations of the external states - this sums over all orderings of the vertex operators with equal weight.

Now consider the four-point function in more detail. It is given by

$$
A_{4}(s, t, u)=\sum_{p e r m s} \int_{0}^{1} d x\left\langle 0\left|S_{k_{1}}^{a_{1}}(0) S_{k_{2}}^{a_{2}}(1) S_{k_{3}}^{a_{3}}(x) S_{k_{4}}^{a_{4}}(\infty)\right| 0\right\rangle=\sum_{\text {perms }} \int_{0}^{1} d x \mathcal{I}(x) \mathcal{J}(x),
$$

where a sum over inequivalent permutations of the order of the external particles is indicated by $\sum_{\text {perms }}$ and

$$
\mathcal{I} \equiv\left\langle 0\left|e^{i k_{1} \cdot X(\infty)} e^{i k_{2} \cdot X(1)} e^{i k_{3} \cdot X(x)} e^{i k_{4} \cdot X(0)}\right| 0\right\rangle=(1-x)^{-t / 2} x^{-s / 2}
$$

(the Mandelstam invariants are defined by $s=-\left(k_{1}+k_{2}\right)^{2}, t=-\left(k_{2}+k_{3}\right)^{2}$ and $u=$ $\left.-\left(k_{1}+k_{3}\right)^{2}\right)$ and

$$
\mathcal{J} \equiv\left\langle 0\left|\mathrm{~J}^{a}(\infty) \mathrm{J}^{b}(1) \mathrm{J}^{c}(x) \mathrm{J}^{d}(0)\right| 0\right\rangle .
$$

The evaluation of $\mathcal{I}$ in (51) involves standard algebra. The current algebra part of the matrix element, $\mathcal{J}$, can be evaluated using,

$$
\left[\mathrm{J}_{n}^{a}, \mathrm{~J}_{m}^{b}\right]=f_{c}^{a b} \mathrm{~J}_{n+m}^{c}+\frac{k}{2} n \delta_{n+m} \delta^{a b}
$$

(where the modes of the currents are defined by $\mathrm{J}^{a}(x)=\sum_{n} \mathrm{~J}_{n}^{a} x^{-n-1}$ ). The current matrix element is given by,

$$
\begin{aligned}
\mathcal{J}(x)= & \sum_{n, m} x^{-m-1}\left\langle 0\left|\mathrm{~J}_{1}^{a} \mathrm{~J}_{n}^{b} \mathrm{~J}_{m}^{c} \mathrm{~J}_{-1}^{d}\right| 0\right\rangle \\
= & \frac{k}{2}\left\{f_{e}^{a b} f^{e c d} x^{-1}(1-x)^{-1}+f_{e}^{a c} f^{e d b}(1-x)^{-1}\right\} \\
& +\frac{k^{2}}{4}\left\{\delta^{a b} \delta^{c d} x^{-2}+\delta^{a c} \delta^{b d}+\delta^{a d} \delta^{b c}(1-x)^{-2}\right\}
\end{aligned}
$$

(the vacuum $|0\rangle$ is annihilated by $\mathrm{J}_{n}^{a}$ for $n \geq 0$ and the expectation value of a single current vanishes). From hereon the level $k$ is to be set equal to 1 . The relations,

$$
\delta^{a b}=\operatorname{tr}\left(T^{a} T^{b}\right), \quad f_{e}^{a b} f^{e c d}=\operatorname{tr}\left(\left[T^{a}, T^{b}\right]\left[T^{c}, T^{d}\right]\right),
$$

will be used to to write the expressions in terms of traces of products of two and four generators of the Lie algebra, $G$.

The full amplitude is given by combining (51) and (54) and summing over permutations with cyclically inequivalent orderings of the vertex operators. The $x$ integrals are simple combinations of Euler beta functions and the result may be expressed as the sum,

$$
A_{4}(s, t, u)=A^{(1)}+A^{(2)}+\text { perms },
$$


where,

$$
A^{(1)}=\frac{\pi^{2}}{2} \operatorname{tr}\left(T^{a_{1}} T^{a_{2}} T^{a_{3}} T^{a_{4}}\right) \frac{u\left(\sin \pi \frac{s}{2}+\sin \pi \frac{t}{2}+\sin \pi \frac{u}{2}\right)}{\Gamma\left(\frac{s}{2}+1\right) \Gamma\left(\frac{t}{2}+1\right) \Gamma\left(\frac{u}{2}+1\right) \sin \left(\pi \frac{s}{2}\right) \sin \left(\pi \frac{t}{2}\right) \sin \left(\pi \frac{u}{2}\right)}
$$

and

$$
A^{(2)}=\operatorname{tr}\left(T^{a_{1}} T^{a_{2}}\right) \operatorname{tr}\left(T^{a_{3}} T^{a_{4}}\right) \frac{\Gamma\left(-\frac{t}{2}+1\right) \Gamma\left(-\frac{u}{2}+1\right)}{4 \Gamma\left(\frac{s}{2}+2\right) \sin \pi \frac{s}{2}}\left(\sin \pi \frac{s}{2}+\sin \pi \frac{t}{2}+\sin \pi \frac{u}{2}\right) .
$$

A sum over cyclic permutations of the external states is indicated. The presence of the tachyon ground state is seen from the ocurrence of the poles in $A^{(2)}$ at $s, t, u=-2$. There are no massless intermediate poles in the amplitude, reflecting the fact that there is no trilinear coupling between the massless scalar states and the photon decouples.

Another way of calculating the amplitudes is by transforming to the cylinder frame and writing the open-string vertex operators in terms of the closed-string variables as in (30), $S_{k}^{a}(\sigma)=K^{a}(\sigma) e^{i k \cdot X(\sigma)}$. The amplitudes may be packaged into the generating functional,

$$
Z[\Phi]=\left\langle 0\left|\exp \left(\int d \sigma \int d^{d} k \Phi^{a}(k) K^{a}(\sigma) e^{i k \cdot X}\right)\right| B\right\rangle .
$$

This gives the same expression for the amplitudes as (49) as can be seen by expanding the exponential in (59) and using $K^{a}(\sigma)|B\rangle \equiv J_{L}^{a}(\sigma)|B\rangle$ together with the current algebra of $J_{L}(\sigma)$. Once all the $K^{a}$ 's have been replaced by $J_{L}^{a}$ 's the boundary state can be replaced by the vacuum state (the bosonic coordinates work in the standard manner). More generally, the closed-string bra state in (59) may be replaced by an arbitrary physical closed-string state, $\langle P|$, to describe the coupling between closed and open strings.

The S-matrix elements for on-shell momenta $k_{1}, \cdots, k_{n}\left(k_{i}^{2}=0\right)$ are given by

$$
A_{n}=\frac{\delta^{n} Z[\Phi]}{\delta \Phi^{a_{1}}\left(k_{1}\right) \cdots \delta \Phi^{a_{n}}\left(k_{n}\right)},
$$

giving,

$$
A_{n}=\Phi_{1}^{a_{1}} \cdots \Phi_{n}^{a_{n}}\left\langle 0\left|\int d \sigma_{1} K^{a_{1}}\left(\sigma_{1}\right) e^{i k_{1} \cdot X\left(\sigma_{1}\right)} \cdots \int d \sigma_{n} K^{a_{n}}\left(\sigma_{n}\right) e^{i k_{n} \cdot X\left(\sigma_{n}\right)}\right| B\right\rangle .
$$

This expression can be written with a particular ordering of the operators along the axis of the cylinder even though the open-string vertices are all attached to the boundary. In other words, the $\sigma$ contours have been displaced by infinitesimal amounts along the cylinder axis to avoid the collision of vertex operators. A precise connection with the previous calculation in the open-string frame involves averaging over the ordering of these operators along the axis of the cylinder. This is equivalent to the principal part prescription implicitly used in evaluating (50). Actually, the result does not generally depend 
on this ordering since the difference between two orderings is a contact term, in which two vertex operators are evaluated at coincident points, that gives vanishing contribution for suitably defined generic external momenta. This method of displacing the integration contours infinitesimally away from the boundary is useful not only because it facilitates the operator algebra but it also acts as an ultraviolet regulator.

\section{4 $G \times G$ symmetry of the $S$-matrix}

We shall now investigate the global symmetries of the S-matrix elements using the formalism based on the cylinder channel operators in which the vertices are proportional to $K^{a}$. The first symmetry is a linear symmetry under $G$ transformations generated by $J_{0}^{a}$. This follows from the identity,

$$
A_{n}=\Phi_{1}^{a_{1}} \cdots \Phi_{n}^{a_{n}}\left\langle 0\left|\int d \sigma_{1} K^{a_{1}}\left(\sigma_{1}\right) e^{i k_{1} \cdot X\left(\sigma_{1}\right)} \cdots \int d \sigma_{n} K^{a_{n}}\left(\sigma_{n}\right) e^{i k_{n} \cdot X\left(\sigma_{n}\right)}\left(1+\epsilon^{a} J_{0}^{a}\right)\right| B\right\rangle,
$$

which is a simple consequence of the fact that $J_{0}|B\rangle=0$. Commuting the factor $J_{0}^{a}$ to the left until it annihilates the closed-string ground state and making use of (48) gives an $n$-particle amplitude with vertex operators transformed so that they have the form (to lowest order in $\epsilon^{a}$ ),

$$
\int d \sigma_{i}\left(\Phi^{a}+f^{a b c} \epsilon^{b} \Phi^{c}\right) K^{a}\left(\sigma_{i}\right) e^{i k \cdot X\left(\sigma_{i}\right)} .
$$

This means that the n-point function is invariant under transformations of the wave functions,

$$
\Phi^{a} \rightarrow \Phi^{a \prime}=\Phi^{a}+f^{a b c} \epsilon^{b} \Phi^{c},
$$

which shows that $J$ acts linearly on the wave functions and is a rotation in the unbroken group $\mathcal{H}=G$.

The $S$-matrix is also invariant under an infinitesimal modification of the boundary state, $|B\rangle_{\eta}=\left(1+\eta^{a} K_{0}^{a}\right)|B\rangle$, which corresponds to an insertion of a zero momentum open string scalar state with wave function $\eta^{a}$. The change in the $n$-particle amplitude is given by,

$$
\delta A_{n}=\Phi_{1}^{a_{1}} \cdots \Phi_{n}^{a_{n}}\left\langle 0\left|\int d \sigma_{1} K^{a_{1}}\left(\sigma_{1}\right) e^{i k_{1} \cdot X\left(\sigma_{1}\right)} \cdots \int d \sigma_{n} K^{a_{n}}\left(\sigma_{n}\right) e^{i k_{n} \cdot X\left(\sigma_{n}\right)} \eta^{a} K_{0}^{a}\right| B\right\rangle .
$$

Commuting $K_{0}$ to the left until it annihilates the closed string vacuum (which is a singlet under $J_{L 0}$ and $J_{R 0}$ separately) and using the commutation relations for $K_{0}$ that follow from (48) leaves terms containing the product of vertices with one vertex replaced by,

$$
\int d \sigma_{i} f^{a b c} \eta^{b} \Phi^{c} J^{a}\left(\sigma_{i}\right) e^{i k \cdot X\left(\sigma_{i}\right)}
$$


Each of these factors can then be commuted to the right until the current density $J^{a}(\sigma)$ annihilates the boundary state. This leaves terms containing commutators of the form,

$$
\left[\int d \sigma_{i} f^{a b c} \eta^{b} \Phi^{c} J^{a}\left(\sigma_{i}\right), \int d \sigma_{j} \Phi^{d} K^{d}\left(\sigma_{j}\right)\right]=\int d \sigma_{i} \int d \sigma_{j} \eta^{b} \Phi^{c} \Phi^{d} f^{a b c} f^{a d e} K^{e}\left(\sigma_{j}\right) \delta\left(\sigma_{i}-\sigma_{j}\right)
$$

(the Schwinger term vanishes because of the antisymmetry of the structure constants $\left.f^{a b c}\right)$. The contribution from the right-hand side vanishes for amplitudes in which the external states have generic momenta. This follows by the usual reasoning that the $e^{i k \cdot X}$ terms in $\mathcal{I}$ give rise to factors of $\left|e^{i \sigma_{i}}-e^{i \sigma_{j}}\right|^{k_{i} k_{j}}$ which multiplies the $\delta\left(\sigma_{1}-\sigma_{2}\right)$ from (67), thereby vanishing (for suitably defined $k_{i} \cdot k_{j}$ ). This means that all the commutators of the form (67) vanish by analytic continuation to physical momenta.

Thus, the scattering amplitude with one zero-momentum Goldstone boson vanishes, which demonstrates the shift symmetry,

$$
\Phi^{a}(x) \rightarrow \Phi^{a \prime}(x)=\Phi^{a}(x)+\eta^{a},
$$

(where $\eta^{a}$ is a constant) characteristic of the nonlinearly realized $\mathcal{G} / \mathcal{H}$ symmetry of cosetspace models.

Although (68) is the symmetry of the S matrix for generic external momenta, the set of nonlinear field transformations seen in the sigma model lagrangian field theory have their counterparts in the S-matrix in processes with non-generic external momenta such that $k_{i} \cdot k_{j}=0$. Here extra contributions arise from the possibility of on-shell intermediate states with $\left(k_{i}+k_{j}\right)^{2}=0$. In the presence of such momentum configurations the $\delta\left(\sigma_{i}-\sigma_{j}\right)$ factor in (67) gives non-vanishing contact terms. In this case the insertion of a zeromomentum boundary scalar state in an $n$-particle amplitude is related to an $n-1$-particle amplitude with a nonlinear transformation on a wave-function, $\delta \Phi^{e}=\eta^{b} \Phi^{c} \Phi^{d} f^{a b c} f^{a d e}$. A more general discussion of the nonlinear symmetries of the model will be given at the end of the next section.

\subsection{Amplitudes with massless photons}

The massless vector open-string state does not arise as an intermediate state in the fourparticle amplitude since all states are neutral under its (Chan-Paton) $U(1)$ charge. Actually, all open-string amplitudes with a single external photon vanish so that none of the massless scalar tree diagrams have intermediate photon poles. However, amplitudes with two or more external photons are nonvanishing. For example, the amplitude with two photons with polarizations $\xi_{r}^{\mu_{r}}\left(k_{r}\right)$ and two massless scalar states is given by,

$$
A_{\gamma \gamma}(s, t, u)=\int \prod_{r=1}^{4} d x_{r} \mu(x) \xi_{1 \mu}\left(k_{1}\right) \xi_{2 \nu}\left(k_{2}\right) \Phi_{3}^{a} \Phi_{4}^{b}\left\langle 0\left|V_{k_{1}}^{\mu}\left(x_{1}\right) V_{k_{2}}^{\nu}\left(x_{2}\right) S_{k_{3}}^{a}\left(x_{3}\right) S_{k_{4}}^{b}\left(x_{4}\right)\right| 0\right\rangle
$$


where the vertex operator for a transverse photon was defined in (31) and $\mu$ denotes the Moebius-invariant measure that can be used to fix three of the $x_{r}$. The amplitude can be written as

$$
A_{\gamma \gamma}(s, t, u)=\int \prod_{i=1}^{4} d x_{i} \mu(x) \frac{\xi_{1} \cdot \xi_{2}}{\left(x_{1}-x_{2}\right)^{2}} \frac{\Phi_{3}^{a} \Phi_{4}^{a}}{\left(x_{3}-x_{4}\right)^{2}} \prod_{i<j}\left(x_{i}-x_{j}\right)^{k_{i} k_{j}}+\cdots .
$$

Only the $\xi_{1} \cdot \xi_{2}$ term is displayed and the $+\cdots$ refers to other contractions, $\xi_{1} \cdot k_{3} \xi_{2} \cdot k_{4}$ etc. (which are determined by gauge invariance). Evaluating the integral and summing over all orderings on the disk gives,

$$
A_{\gamma \gamma}=\xi_{1} \cdot \xi_{2} \Phi_{3}^{a} \Phi_{4}^{a} \frac{\Gamma(-u / 2+1) \Gamma(-t / 2+1)}{\Gamma(s / 2+2) \sin (\pi s / 2)}\{\sin (\pi s / 2)+\sin (\pi t / 2)+\sin (\pi u / 2)\}+\cdots
$$

\section{Low energy limit and principal chiral models}

Since the tree-level $S$ matrix for the massless scalars has the symmetries associated with coset space nonlinear sigma models its low-energy limit should simply be such a model. In order to make this explicit we shall now consider the low energy properties of the theory with boundary condensates. This is a description in terms of the massless degrees of freedom at energies such that all the massive string states are effectively integrated out.

Recall that the coset model with $\mathcal{G}=G \times G$ and $\mathcal{H}=G$ is equivalent to the principal chiral sigma model in which the scalar fields parameterize the group manifold of $G$. The Goldstone boson ('pion') fields that arise in principal chiral models parameterize the group manifold $G$, so that a group element is given by $g(X)=\exp \left(\Phi^{a}(X) T^{a}\right)$. The action of the principal chiral model,

$$
\int d^{D} x \frac{1}{2} \operatorname{tr}\left(g^{-1} \partial_{\mu} g g^{-1} \partial^{\mu} g\right)
$$

has global invariance under left and right multiplication with group elements $\rho_{L}$ and $\rho_{R}$.

We will first indicate how the low-energy effective action can be deduced by comparing the low-energy behaviour of the $n$-particle $S$-matrix elements to terms in the expansion in powers of $\Phi$ of conventional principal chiral nonlinear sigma models. Since $g$ has an infinite expansion in powers of $\Phi$ it is complicated to carry this out systematically beyond the lowest orders. We will instead consider condition for the vanishing of the one loop beta function that is the lowest-order requirement of conformal invariance will be seen to be the familiar equations of motion for the principal chiral models based on the group $G$. 


\subsection{Low energy limit of S-matrix elements}

The low energy limit of the four point amplitude can be obtained from (56), (57) and (58) by expanding the expressions in powers of $s, t$ and $u$. This corresponds to an expansion in powers of $\alpha^{\prime}$. The leading terms come from (57), using $s+t+u=0$ and

$$
\sin \frac{\pi \alpha^{\prime} s}{2}+\sin \frac{\pi \alpha^{\prime} t}{2}+\sin \frac{\pi \alpha^{\prime} u}{2}=-\frac{\pi^{3}}{16} \alpha^{\prime 3} s t u+o\left(\alpha^{\prime 5}\right)
$$

the contribution from $(57)$ is,

$$
A^{(1)}=-\frac{\pi^{2}}{2} \operatorname{tr}\left(T^{a_{1}} T^{a_{2}} T^{a_{3}} T^{a_{4}}\right) u+\text { perms }+o\left(\alpha^{\prime 2}\right),
$$

while the contribution from $(58)$ is

$$
A^{(2)}=-\frac{\pi^{2}}{32} \operatorname{tr}\left(T^{a_{1}} T^{a_{2}}\right) \operatorname{tr}\left(T^{a_{3}} T^{a_{4}}\right) \alpha^{\prime} t u+\text { perms }+o\left(\alpha^{\prime 3}\right) .
$$

Therefore, in the low-energy limit the dominant contribution to the four-particle amplitude comes from $A^{(1)}$. This is just the form of the amplitude expected for a nonlinear sigma model since it is linear in the Mandelstam invariants which means that it comes from a lagrangian with two space-time derivatives. More precisely, the amplitude is reproduced by an action of the form,

$$
\int d^{D} x\left(\frac{1}{2} \operatorname{tr}\left(\partial_{\mu} \Phi \partial^{\mu} \Phi\right)+\operatorname{tr}\left(\Phi \partial_{\mu} \Phi \Phi \partial^{\mu} \Phi\right)-\operatorname{tr}\left(\Phi \Phi \partial_{\mu} \Phi \partial^{\mu} \Phi\right)\right)
$$

(where $\Phi \equiv \Phi^{a} T_{i j}^{a}$ ) which are the lowest-order terms in the expansion of the action (72) of the principal chiral model. In order to verify that the leading $\alpha^{\prime}$ terms in the lowenergy limit are identical to those of the principal chiral model it is necessary to compare amplitudes with arbitrary numbers of external Goldstone bosons with the amplitudes derived from the $\Phi$ expansion of the field theory lagrangian. There are generally intermediate massless poles in multi-particle amplitudes (a feature that does not arise in the four-particle amplitude) which must be subtracted explicitly before taking the low-energy limit, which complicates the procedure.

If the open-string photon is ignored the higher-order terms in the $\alpha^{\prime}$ expansion of the massless scalar amplitudes give higher-derivative generalizations of the nonlinear sigma model. For example, the terms of order $\alpha^{\prime}$ relative to the leading terms correspond to a model with four derivatives. However, the presence of the photon affects the terms beyond lowest-order in $\alpha^{\prime}$.

The observation that a single photon does not couple to any number of massless Goldstone bosons is in accord with the fact that there is no action linear in the abelian field strength $F_{[\mu \nu]}$ coupling to a function of the group element, $g=e^{\Phi \cdot T}$. The simplest 
candidate for a covariant coupling betwen photons and the massless scalars is the linear combination of two terms,

$$
C\left(F_{[\mu \nu]} F^{[\nu \rho]} \operatorname{tr}\left(g^{-1} \partial_{\rho} g g^{-1} \partial^{\mu} g\right)+\alpha F_{[\mu \nu]} F^{[\nu \mu]} \operatorname{tr}\left(g^{-1} \partial_{\rho} g g^{-1} \partial^{\rho} g\right)\right)
$$

which gives amplitudes with two photons. The constant $\alpha$ is undetermined in the lowenergy field theory but may be determined by comparison with the low-energy limit of the string theory amplitudes. The low energy limit of the string amplitude is obtained by expanding (71) in the Mandelstam invariants,

$$
A_{\gamma \gamma}=\xi_{1} \cdot \xi_{2} \Phi_{3}^{a} \Phi_{4}^{a} \alpha^{\prime} \frac{\pi^{2}}{8} u t+\cdots+o\left(\alpha^{\prime 3}\right)
$$

On the other hand the contribution of the two terms in (77) to the four-particle amplitude,

$$
A_{\gamma \gamma}=\xi_{1} \cdot \xi_{2} \Phi_{3}^{a} \Phi_{4}^{a} C\left(u^{2}+t^{2}-2 \alpha s^{2}\right)+\cdots
$$

Comparison of this expression with (78) uniquely determines $\alpha=\frac{1}{2}$ (and $C$ is an irrelevant overall normalization).

\subsection{Equation of motion for principal chiral models}

Before discussing the condition for the vanishing of the one-loop beta function we shall review the form of the equations of motion of the principal chiral models in terms of the field definitions that arise in the boundary condensate. The variation of the lagrangian (72) under the infinitesimal variation $\delta g=g \kappa$ is,

$$
\delta L=\operatorname{tr}\left(\partial_{\mu}\left(g^{-1} \partial^{\mu} g\right) \kappa\right)
$$

so that the equation of motion has the form

$$
\partial_{\mu} A^{\mu}=\partial_{\mu}\left(g^{-1} \partial^{\mu} g\right)=0
$$

where $A^{\mu}=g^{-1} \partial^{\mu} g$ is a flat non-abelian gauge potential. The equation of motion (81) can be written as an equation for $\Phi^{a}$ by making use of the identity,

$$
A^{\mu}=\sum_{n=0}^{\infty} \frac{(-1)^{n}}{(n+1) !}\left(A d_{\Phi}\right)^{n} \partial^{\mu} \Phi,
$$

where $A d_{C} D=[C, D]$ (and $C, D$ are matrices) so that,

$$
\left(A d_{C}\right)^{n} D=[C,[C,[C, \cdots,[C, D] \cdots]]]
$$


( $\cdots$ indicates that there are $n C$ 's in the expression). Applying another derivative gives the equation of motion,

$$
\partial_{\mu} A^{\mu}=\sum_{n=0}^{\infty} \frac{(-1)^{n}}{(n+1) !}\left\{\left(A d_{\Phi}\right)^{n} \partial^{2} \Phi+\sum_{k=0}^{n-1}\left(A d_{\Phi}\right)^{k}\left(A d_{\partial^{\mu} \Phi}\right)\left(A d_{\Phi}\right)^{n-k-1} \partial^{\mu} \Phi\right\}=0 .
$$

These expressions are more familiar when written in terms of the metric on the group manifold, $\gamma_{a b}\left(\Phi^{c}\right)$, with the action density (72) given by $\frac{1}{2} \gamma_{a b} \partial_{\mu} \Phi^{a} \partial^{\mu} \Phi^{b}$, in which case the equation of motion becomes,

$$
\gamma_{a b} \partial^{2} \Phi^{b}+\frac{\partial \gamma_{a b}}{\partial \Phi_{c}} \partial_{\mu} \Phi^{c} \partial^{\mu} \Phi^{b}-\frac{1}{2} \frac{\partial \gamma_{c b}}{\partial \Phi_{a}} \partial_{\mu} \Phi^{c} \partial^{\mu} \Phi^{b}=0
$$

\subsection{The one-loop beta function and the $\Phi$ equations of motion}

The total action for a space-time dependent boundary condensate is,

$$
S=S_{0}+S_{B}
$$

where $S_{0}$ is the bulk term and the boundary term is given by,

$$
S_{B}=\frac{1}{2} \int d \sigma \Phi^{a}(X(\sigma))\left(J_{L}^{a}(\sigma)+J_{R}^{a}(\sigma)\right)
$$

where $J_{L}^{a}(\sigma)=\psi_{L}^{i}(\sigma) T_{i j}^{a} \psi_{L}^{j}(\sigma)$ and $J_{R}^{a}(\sigma)=\psi_{R}^{i}(\sigma) T_{i j}^{a} \psi_{R}^{j}(\sigma)$. We shall use a background field method in which the space-time coordinates $X^{\mu}$ are written as the sum of a constant classical part $\left(\tilde{X}^{\mu}\right)$ and a quantum fluctuation $\left(\xi^{\mu}\right)$,

$$
X(\sigma)=\tilde{X}+\xi(\sigma)
$$

The background-dependent coupling $\Phi(X(\sigma))$ is expanded in powers of $\xi$,

$$
\Phi^{a}(X)=\Phi^{a}(\tilde{X})+\partial_{\mu} \Phi^{a}(\tilde{X}) \xi^{\mu}+\frac{1}{2} \partial_{\mu} \partial_{\nu} \Phi^{a}(\tilde{X}) \xi^{\mu} \xi^{\nu}+o\left(\xi^{3}\right) .
$$

Since each $\xi$ comes with a space-time derivative on the background field $\Phi$ and we are interested in the low energy or 'small curvature' limit of the theory only a small number of derivatives need to be considered to extract the leading $\alpha^{\prime}$ dependence. We shall keep only terms with up to two derivatives. Using the expansion (89) in the boundary action, $S_{B}$, gives vertices with two fermion lines and arbitrary number of $\xi$ lines. The fermions and $\xi$ propagators evaluated between points on the boundary are derived from the bulk part of the action and satisfy the usual boundary conditions (i.e., Neumann boundary conditions on $\xi$ ). 
We will calculate the one-loop counterterm bilinear in the fermion fields that renormalizes the value of $\Phi$ in $S_{B}$. This has the form, $\int d \sigma \psi^{i}(\sigma) \Gamma_{i j}(\Phi) \psi^{j}(\sigma)$. If the quantum corrections preserve the property that the theory is conformally invariant the overall coefficient of the logarithmically divergent contributions (which signal a breakdown of scale invariance) must vanish which means that the renormalization group beta function of the scalar field must vanish for the background condensate to define a conformal quantum field theory. This is equivalent to ensuring that the theory is BRST invariant by imposing the condition,

$$
Q_{B R S T} e^{\int d \sigma \Phi^{a}(X(\sigma)) J_{L}^{a}(\sigma)}|B\rangle=0,
$$

where $Q_{B R S T}$ is the bulk BRST charge. In the Siegel gauge this is equivalent to imposing the energy-momentum tensor conditions, (14). However, the fact that the boundary state satisfies this condition is not by itself sufficient to guarantee BRST invariance due to possibility of an anomaly that generates a cosmological constant as argued by Fischler and Susskind [30], [28], 227] (see section 5).

A priori, infinitely many diagrams contribute to this divergence (even to lowest nontrivial order in $\alpha^{\prime}$, which involves a contraction of two $\xi^{\prime}$ 's) because of the presence of the constant term, $\Phi^{a}(\tilde{X}) \psi^{i}(\sigma) T_{i j}^{a} \psi^{j}(\sigma)$ which is quadratic in the fermion fields. It is sensible to eliminate this term in order to reduce the one-loop problem to the evaluation of a single diagram.

It is convenient to use the operator realization of the string path integral by quantizing on equal $\tau$ slices on the semi-infinite cylinder (where $\tau$ is the axis of the cylinder). The currents $K=\left(J_{L}-J_{R}\right) / 2$ may be replaced by the left-movers $J_{L}$ using the fact that the boundary state, $|B\rangle$, relates right-movers to left-movers and the partition function is given by,

$$
\begin{aligned}
& Z[\Phi]=\left\langle P\left|\exp \int d \sigma \Phi^{a}(X(\sigma)) K^{a}(\sigma)\right| B\right\rangle \\
& =\left\langle P\left|\exp \left(\int d \sigma\left\{\Phi^{a}(\tilde{X})+\partial_{\mu} \Phi^{a}(\tilde{X}) \xi^{\mu}+\frac{1}{2} \partial_{\mu} \partial_{\nu} \Phi^{a}(\tilde{X}) \xi^{\mu} \xi^{\nu}+o\left(\xi^{3}\right)\right\} J_{L}^{a}(\sigma)\right)\right| B\right\rangle(91)
\end{aligned}
$$

where the presence of the state $\langle P|$ again allows for the coupling of the boundary to an arbitrary closed-string state.

In order to evaluate this expression in perturbation theory the exponent is written as a sum of two pieces, $C$ and $D$, where

$$
C=\int d \sigma \Phi^{a}(\tilde{X}) J_{L}^{a}(\sigma)=\Phi^{a}(\tilde{X}) J_{L 0}^{a},
$$

is independent of $\xi$, while the second involves all powers of $\xi$,

$$
D=\int d \sigma\left\{\partial_{\mu} \Phi^{a}(\tilde{X}) \xi^{\mu}+\frac{1}{2} \partial_{\mu} \partial_{\nu} \Phi^{a}(\tilde{X}) \xi^{\mu} \xi^{\nu}+o\left(\xi^{3}\right)\right\} J_{L}^{a}(\sigma) .
$$


The term $C$ is the constant piece of the exponent (91) and can be moved to the left with the help of Baker-Campbell-Hausdorf formula,

$$
e^{C+D}=e^{C} e^{\sum_{r=0, s=1}^{\infty} H_{r, s}(C, D)} .
$$

Here the $H_{r, s}(C, D)$ are homogeneous polynomials of degree $r$ in $C$ and $s$ in $\mathrm{D}$ which are made out of multiple commutators of $C$ and $D$ The partition function may be written in terms of the $H_{n, m}$ 's,

$$
Z[\Phi]=\left\langle P\left|e^{C} e^{\sum_{r=0, s=1}^{\infty} H_{r, s}(C, D)}\right| B\right\rangle .
$$

The factor of $e^{C}=e^{\Phi^{a}(\tilde{X}) J_{L 0}^{a}}$ simply transforms the closed-string bra state and can be ignored. We will see that the one-loop logarithmic divergences come only from terms in $H_{n, 1}$ and $H_{n, 2}$. These terms can be obtained by a power series expansion of (94) and are given (after some algebra) by,

$$
H_{n, 1}=\frac{(-1)^{n}}{(n+1) !}\left(A d_{C}\right)^{n} D
$$

and

$$
H_{n, 2}=\frac{1}{2} \frac{(-1)^{n}}{(n+2) !} \sum_{k=0}^{n-1}\left(A d_{C}\right)^{k} A d_{D}\left(A d_{C}\right)^{n-k} D .
$$

As noted above we are interested in terms with up to two space-time derivatives or equivalently two powers of the quantum fluctuations, $\xi$. There are two contributions that we need to consider. Firstly, $H_{n, 1}$ with arbitrary $n$ contains terms of the form $\partial_{\mu} \partial_{\nu} \Phi(\tilde{X}) \xi^{\mu} \xi^{\nu}$ coming from $D$. The expression in (96) can be simplified by using the fact that $C$ is proportional to the zero mode of the current so its commutation relations simply reduce to group theoretic commutators, as can be seen from the general expression,

$$
\begin{aligned}
A d_{C} \chi=\left[\Phi^{a}(\hat{X}) J_{L 0}^{a}, \int d \sigma \chi^{b}(\sigma) J_{L}^{b}(\sigma)\right] & =\int d \sigma f^{a b c} \Phi^{a}(\hat{X}) \chi^{b}(\sigma) J_{L}^{c}(\sigma) \\
& =\int d \sigma J_{L}^{a}(\sigma) A d_{\Phi} \chi^{a}(\sigma),
\end{aligned}
$$

where $\chi^{a}(\sigma)$ is an arbitrary matrix-valued function and the second line makes use of the definition of $A d_{C} D^{a}$,

$$
A d_{C} D^{a}=\left(A d_{C} D\right)_{i j} T_{i j}^{a} .
$$

Denoting the terms in the sum in (96) (with $\mathrm{C}$ defined by (92) and $D$ defined by (93)) that can lead to logarithmic one-loop divergences by $H_{n, 1}^{\prime}$ we find by iterating (98),

$$
H_{n, 1}^{\prime}=\frac{(-1)^{n}}{(n+1) !} \int d \sigma J_{L}^{a}(\sigma)\left(A d_{\Phi}\right)^{n}\left\{\frac{1}{2} \partial_{\mu} \partial_{\nu} \Phi^{a}(\tilde{X}) \xi^{\mu}(\sigma) \xi^{\nu}(\sigma)+\partial_{\mu} \Phi^{a}(\tilde{X}) \xi^{\mu}(\sigma)\right\}
$$


The terms $H_{n, 2}$ defined by (97) are quadratic in $D$ so they give rise to new terms that are quadratic in $\xi$ that arise as bilinears in $D^{\prime}=\int d \sigma \partial_{\mu} \Phi^{a} J_{L}^{a} \xi^{\mu}$. As before, terms of the form $\left(A d_{C}\right)^{k} \chi$ in (97) are given by the group theory that follows from (98). However, the factors of the form $A d_{D}\left(A d_{C}\right)^{n-k} D$ involve a commutator of two terms which are linear in both $\xi$ and in the current densities. This commutator is given by,

$$
\begin{gathered}
{\left[\int d \sigma_{1} J_{L}^{a}\left(\sigma_{1}\right) \partial_{\mu} \Phi^{a} \xi^{\mu}\left(\sigma_{1}\right), \int d \sigma_{2} J_{L}^{b}\left(\sigma_{2}\right)\left(A d_{\Phi}\right)^{k_{2}} \partial_{\nu} \Phi^{b} \xi^{\nu}\left(\sigma_{2}\right)\right]} \\
=\int d \sigma_{1} \partial_{\mu} \Phi^{a} \xi^{\mu}\left(\sigma_{1}\right) \int d \sigma_{2}\left(A d_{\Phi}\right)^{k_{2}} \partial_{\nu} \Phi^{b} \xi^{\nu}\left(\sigma_{2}\right) \\
\left\{f^{a b c} J_{L}^{c}\left(\sigma_{1}\right) \delta\left(\sigma_{1}-\sigma_{2}\right)+\delta^{a b} \delta^{\prime}\left(\sigma_{1}-\sigma_{2}\right)\right\} .
\end{gathered}
$$

The terms on the right-hand side come from the equal time commutators of the current densities, $J_{L}$. The first term is,

$$
\int d \sigma J_{L}^{a}(\sigma) A d_{\partial_{\mu} \Phi}\left(A d_{\Phi}\right)^{k_{2}} \partial_{\nu} \Phi^{a} \xi^{\mu}(\sigma) \xi^{\nu}(\sigma)
$$

while the $\delta^{\prime}$ term in (101) gives $\int d \sigma \partial_{\mu} \Phi^{a}\left(A d_{\Phi}\right)^{k_{2}} \partial_{\nu} \Phi^{b} \delta^{a b} \xi^{[\mu}(\sigma) \partial_{\sigma} \xi^{\nu]}(\sigma)$, which will not lead to a logarithmic divergence. As a result, the terms in $H_{n, 2}$ that can lead to one-loop divergences (denoted by $H_{n, 2}^{\prime}$ ) are given by,

$$
H_{n, 2}^{\prime}=\frac{(-1)^{n+1}}{2(n+2) !} \int d \sigma J_{L}^{a}(\sigma) \sum_{k=0}^{n-1}\left(A d_{\Phi}\right)^{k} A d_{\partial_{\mu} \Phi}\left(A d_{\Phi}\right)^{n-k} \partial_{\nu} \Phi^{a} \xi^{\mu}(\sigma) \xi^{\nu}(\sigma) .
$$

The modified action given by $H_{n, 1}+H_{n, 2}$ may now be used to calculate the logarithmic divergent graphs, given by the following diagram,

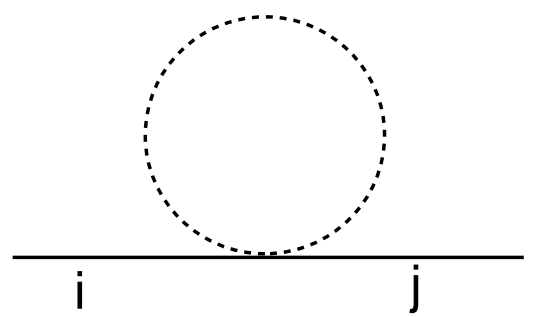

The vertex is the $\xi \xi$ term in $H_{n, 1}^{\prime}$ or $H_{n, 2}^{\prime}$ in (100) and (103), respectively (the dashed line indicates the $\xi$ propagator and the full lines $\psi$ propagators). In evaluating the matrix element (91) the logarithmically divergent self-contraction of $\xi^{\mu}(\sigma) \xi^{\nu}(\sigma)$ at coincident points requires regularization by a cut-off $\Lambda$ defined by $\left\langle\xi^{\mu}(\sigma) \xi^{\nu}(\sigma)\right\rangle=G^{\mu \nu}(\sigma, \sigma)=\delta^{\mu \nu} \ln \Lambda$. The contributions from (100) and (103) are,

$$
a_{1}=J_{L 0}^{a} \sum_{n} \frac{(-1)^{n}}{2(n+1) !}\left(A d_{\Phi}\right)^{n} \partial_{\mu} \partial^{\mu} \Phi^{a} \ln \Lambda .
$$


and

$$
a_{2}=J_{L 0}^{a} \sum_{n} \frac{(-1)^{n+1}}{2(n+2) !} \sum_{k=0}^{n-1} \int d \sigma\left(A d_{\Phi}\right)^{k} A d_{\partial_{\mu} \Phi}\left(A d_{\Phi}\right)^{n-k} \partial^{\nu} \Phi^{a} \ln \Lambda .
$$

In this discussion the presence of the term linear in $\xi$ in $H_{n, 1}^{\prime}$ (100) has so far been ignored. This is a vertex with a single $\xi$ that leads to the following one-loop diagram, which is easily seen to vanish,

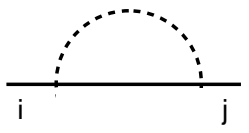

The final result for the logarithmically divergent one-loop counterterm, $\Gamma$, is given by $a_{1}+a_{2}$. The vanishing of the beta function, $\beta^{a}=\Lambda^{-1} d \Gamma^{a} / d \Lambda=0$, gives the equation of motion,

$$
\sum_{n}\left\{\frac{(-1)^{n}}{2(n+1) !}\left(A d_{\Phi}\right)^{n} \partial^{2} \Phi^{a}+\frac{(-1)^{n+1}}{2(n+2) !} \sum_{k=0}^{n-1}\left(A d_{\Phi}\right)^{k} A d_{\partial_{\mu} \Phi}\left(A d_{\Phi}\right)^{n-k} \partial^{\nu} \Phi^{a}\right\}=0
$$

which is just the equation of motion of the principal chiral model given in (84), which is equivalent to (85).

As a corollary we can now return to the discussion of the nonlinear transformations described in the last section. These nonlinear transformations can be deduced from the partition function, (91), by considering the effect of shifting the $\Phi$ field (now taken to satisfy the beta function equation) in the exponent of the partition function to $\Phi^{\prime}(X(\sigma))=\Phi^{a}(X(\sigma))+\eta^{a}$ (with constant $\eta$ ) and using (94) with $C=\eta^{a} J_{L o}^{a}$ and $D=\int \Phi^{a}(X(\sigma)) J_{L}^{a}(\sigma)$. For purely open-string processes, in which $\langle P|=\langle 0|$ in (91), the factor of $e^{C}=e^{\eta^{a} J_{L 0}^{a}}$ is equivalent to unity on the external closed-string bra state giving,

$$
Z\left[\Phi^{\prime}\right]=\left\langle 0\left|\exp \int d \sigma \sum_{r=0, s=1}^{\infty} H_{r, s}(C, D)\right| B\right\rangle
$$

(more generally, the closed-string bra state transforms from $\langle P|$ to $\langle P| e^{\eta^{a} J_{L 0}^{a}}$ ). We are interested in the terms up to linear order in $\eta$ in the remaining exponent. The term independent of $\eta$ is $H_{0,1}=D$ (all $H_{0, n}$ with $n>1$ vanish) while $H_{1, n}$ are the terms linear in $\eta$ involving $n$ powers of $\Phi$. It is easy to verify that $H_{1,1}=\frac{1}{2}[C, D]$ and $H_{1,2}=$ $-[D,[D, C]] / 12$ so that the exponent of $(107)$ can be written as $\int d \sigma \Phi^{\prime \prime a} J_{L}^{a}(\sigma)$ where,

$$
\Phi^{\prime \prime}(\sigma)=\frac{1}{2} f^{a b c} \eta^{b} \Phi^{c}(X(\sigma))+\frac{1}{12} f^{a b e} f^{e c d} \eta^{c} \Phi^{b}(X(\sigma)) \Phi^{d}(X(\sigma))+o\left(\Phi^{3}\right) .
$$

Thus, the theory is invariant under the nonlinear transformation $\Phi \rightarrow \Phi^{\prime}=\Phi+\eta-\Phi^{\prime \prime}$. 


\section{$5 \quad$ Mixing of open and closed strings}

The quantum loop corrections to the tree-level theory generate the coupling between open and closed strings. It has long been appreciated [21] that the mixing of open and closed-string states in the Minkowski space theory gives rise to a mechanism whereby the closed-string antisymmetric tensor potential $\left(B_{[\mu \nu]}\right)$ gains a mass by absorbing the massless neutral open-string vector state (with potential $A_{\mu}$ ). The effective action for the massive state can be written as

$$
\int d^{D} x\left(\frac{1}{6} d B^{2}+\frac{1}{2}(\mathrm{~g} B-F)^{2}\right)
$$

where $F=d A$ and $\mathrm{g}$ is the open-string coupling constant. The modified gauge symmetry of this action is $\delta B=d \xi, \delta A=d \Lambda+\mathrm{g} \xi$, where $\Lambda$ is a scalar and $\xi$ is a one-form. This can be used to define a gauge in which $A$ is eliminated, resulting in the lagrangian for a massive antisymmetric potential. In four dimensions the antisymmetric tensor is equivalent to a scalar state defined by $d \phi=* d B$ and (109) displays the usual abelian Higgs mechanism (in the nonlinear limit in which the mass of the Higgs particles are infinite). The terms involving non-zero powers of $\mathrm{g}$ arise from a world-sheet with the topology of a disk. There is an interaction between the closed-string state, $B$, on the interior of the disk and the open-string state, $A$, on the boundary. The $O\left(\mathrm{~g}^{2}\right)$ mass term for $B$ comes from the coupling of two $B$ states on the interior of the disk. In addition, the dilaton has a non-zero coupling to the vacuum via its coupling to the boundary of the disk. This generates a non-zero cosmological constant by the Fischler-Susskind mechanism [30]. This phenomenon may be associated with anomaly that comes from contact terms that arise in proving the BRST invariance of amplitudes (28] 27]). These contact terms can be ascribed to the presence of a zero-momentum dilaton and trace of the graviton in the closed-string channel that couples to the disk boundary.

Since the closed-string sector carrying the gauge symmetry $G \times G$ at the enhanced symmetry points couples linearly to the coset-space open-string states it is to be expected that (109) should generalize to a non-abelian Higgs mechanism. The coset-space currents, $K^{a}$, correspond to a linear combination of gauge bosons that should gain a mass by swallowing the massless open-string scalar states.

The mixing of the coset-space pions with massless closed-string gauge fields is seen from the non-vanishing of $\mathrm{g}\left\langle\right.$ phys $\left.\left|\oint d \sigma K^{a}(\sigma) e^{i k \cdot X(\sigma)}\right| B\right\rangle$ (where $\langle$ phys $|$ is a physical closedstring gauge particle state and we have explicitly included a factor of the open-string coupling to illustrate that the pion fields are of order g). Furthermore, the fact that the theory contains the requisite gauge invariance can be seen directly from the bulk world-sheet action, in which the target-space gauge fields occur in the terms,

$$
\int d^{2} z\left(\psi_{L}\left(\partial+A_{L \mu} \cdot \partial X^{\mu}\right) \psi_{L}+\psi_{R}\left(\bar{\partial}+A_{R \mu} \cdot \bar{\partial} X^{\mu}\right) \psi_{R}\right)
$$


where $A_{L \mu}=A_{L \mu}^{a} T_{i j}^{a}$ and $A_{R \mu}=A_{R \mu}^{a} T_{i j}^{a}$ are the background gauge potentials. In the absence of boundaries conformal invariance requires these potentials to satisfy the YangMills field equations [31], 32], 33]. The target-space theory is invariant under the gauge transformations,

$$
A_{L} \rightarrow A_{L}^{\prime}=\rho_{L}^{-1} A_{L} \rho_{L}-\rho_{L}^{-1} d \rho_{L}, \quad A_{R} \rightarrow A_{R}^{\prime}=\rho_{R}^{-1} A_{R} \rho_{R}-\rho_{R}^{-1} d \rho_{R}
$$

where $\rho_{L}=\rho_{L}^{a} T_{i j}^{a}=e^{\Lambda_{L}}$ and $\rho_{R}=\rho_{R}^{a} T_{i j}^{a}=e^{\Lambda_{R}}$ are the gauge parameters for $G \times G$. This can be seen by substituting (111) in (110) and noting that the $\rho_{L}$ and $\rho_{R}$ dependence can be eliminated by redefining the world-sheet fermion fields by

$$
\psi_{L} \rightarrow \psi_{L}^{\prime}=\rho_{L} \psi_{L}, \quad \psi_{R} \rightarrow \psi_{R}^{\prime}=\rho_{R} \psi_{R}
$$

In the presence of boundaries this redefinition of the fermion fields affects the boundary condensate because it changes the relation between the left-moving and right-moving fermions at the boundary. The new boundary state must satisfy (dropping the primes),

$$
\left(\psi_{R}-i \rho_{R} \rho_{L}^{-1} \psi_{L}\right)|B\rangle_{\rho}=0
$$

where $\rho_{L}$ and $\rho_{R}$ are here functions of the boundary values of $X(\sigma, \tau)$. The boundary state satisfying these conditions can be expressed in terms of the Lie-algebra valued functions, $\Lambda_{L}$ and $\Lambda_{R}$, by

$$
|B\rangle_{\rho}=\exp \int d \sigma\left(\psi_{L}^{i}(\sigma) \Lambda_{L i j}(X(\sigma)) \psi_{L}^{j}(\sigma)-\psi_{R}^{i}(\sigma) \Lambda_{R i j}(X(\sigma)) \psi_{R}^{j}(\sigma)\right)|B\rangle .
$$

For infinitesimal $\Lambda=\Lambda_{L}-\Lambda_{R}$ it is clear that the dependence on the gauge parameters can be eliminated by shifting the scalar field in the boundary action,

$$
\Phi \cdot T \rightarrow \Phi \cdot T+\Lambda_{L}-\Lambda_{R}
$$

which is a shift of the scalar fields in the coset $\left(G_{L} \times G_{R}\right) / G_{\text {diag }}$.

This shift of the scalar fields is characteristic of the non-abelian Higgs phenomenon. The low-energy action for the massive gauge fields embodying this gauge symmetry is,

$$
\int d^{D} x\left(\frac{1}{4} \operatorname{tr} F^{2}+\frac{\mathrm{g}^{2}}{2} \operatorname{tr}\left(A^{K}+\frac{1}{\mathrm{~g}^{2}} g^{-1} d g\right)^{2}\right),
$$

where $F$ is the non-abelian field strength for the $\operatorname{dim}(G)+\operatorname{dim}(G)$ gauge bosons in $G \times$ $G$ and $A^{K}=\left(A_{L}-A_{R}\right) / 2$ are the components in the coset directions in the algebra. The dependence on the open-string coupling constant is consistent with the conventional normalization in which $\Phi$ (where $g=e^{\Phi}$ ) is of order $g$. These scalar fields can be eliminated by a suitable gauge choice in the usual manner. The equations of motion that follow from 
this action should be derived directly as the condition for conformal invariance of the coupled bulk and boundary system.

The presence of the gauge field mass term in (116) originates from the coupling of two massless vector states to the disk, $\mathrm{g}^{2} \int d z d z^{\prime}\left\langle V_{ \pm}^{a \mu}(z) V_{ \pm}^{b \nu}\left(z^{\prime}\right)\right\rangle$ (where $\left.V_{ \pm}^{a \mu}=V^{a \mu} \pm \tilde{V}^{a \mu}\right)$. In the frame in which the disk is represented by a semi-infinite cylinder this can be expressed as the matrix element,

$A_{ \pm}^{\mu \nu a b}=\mathrm{g}^{2}\left\langle k\left|\left(\alpha_{1}^{\mu} J_{R 1}^{a} \pm \tilde{\alpha}_{1}^{\mu} J_{L 1}^{a}\right) \int_{0}^{2 \pi} d \sigma \sum_{n, m}\left(\alpha_{n}^{\nu} J_{R m}^{b} \pm \tilde{\alpha}_{-n}^{\nu} J_{L-m}^{a}\right) e^{-i k X(\sigma)} e^{i(n-m) \sigma} \int \frac{d q}{q^{3-N}}\right| B\right\rangle$

where the length of the cylinder (the radius of the disk) is the one real modular parameter for this process. This expression is divergent due to the tachyon in the cylinder channel. Regulating this in a gauge-invariant manner leads to mass terms for the antisymmetric combinations of gauge fields (with vertex operators $V_{-}^{a \mu}$ ) while the gauge fields in the unbroken diagonal group $\left(V_{+}^{a \mu}\right)$ remain massless.

Thus, at the enhanced symmetry point half the gauge fields get masses of order $\mathrm{g}^{2}$ by absorbing the massless open-string scalars lying in the $G \times G / G$ coset space, leaving $\operatorname{dim}(G)$ massless gauge fields in the diagonal subgroup.

However, the complete analysis is complicated by the fact that the massless closedstring scalars (the moduli) also couple to the disk and may gain mass. Their masses are determined by the two-point functions,

$$
\left\langle\Phi^{a b} \Phi^{c d}\right\rangle=\int\left\langle J_{L}^{a}\left(z_{1}\right) J_{R}^{b}\left(\bar{z}_{1}\right) J_{L}^{c}\left(z_{2}\right) J_{R}^{d}(\bar{z})\right\rangle_{d i s k},
$$

where $\Phi^{a b}$ denotes the closed-string scalars with vertex operators $J_{L}^{a} J_{R}^{b} e^{i k X}$. Among several non-zero contributions this expression possesses a divergence due to the coupling of a massless state to the boundary of the disk which has the form,

$$
\left\langle\Phi^{a b} \Phi^{c d} \Phi^{e f}\right\rangle_{\text {sphere }} \Delta\left\langle\Phi^{e f}\right\rangle_{\text {disk }},
$$

where $\Delta \sim \int d q / q \sim \ln \Lambda$. The three-point function on the sphere has the form $f^{a c e} f^{b d f}$ and the one-point function on the disk is proportional to $\delta^{e f}$ so the divergence is due to the exchange of the trace $\Phi^{e e}$. This is similar to the divergence associated with the massless dilaton [30], [28], [27] and can be cancelled by adding a cut-off dependent condensate to the classical theory which modifies the action by the addition of a term,

$$
S=S_{0}+g \int d^{2} z J_{L}^{e} J_{R}^{e} \ln \Lambda
$$

which corresponds to a non-conformal perturbation of the classical theory. There are additional mass terms in (118) which give finite masses to $\Phi^{e e}$ as well as to the antisymmetric scalar fields, $\Phi^{[a b]}$. 


\section{Nonorientable world-sheets}

We now turn to a brief discussion of theories defined on non-orientable world-sheets. In such theories the closed-string sector space of states is projected onto the subspace in which the 'twist' operator, $\Omega$ (that interchanges the left-moving and right-moving spaces), has eigenvalue +1 . This means that $(1-\Omega) \mid$ phys $\rangle=0$, where $\mid$ phys $\rangle$ is a physical closedstring state. This projection eliminates the antisymmetric tensor field and half the $G \times G$ gauge fields, leaving those in the diagonal $G$ subgroup. Similarly $\operatorname{dim}(G)$ massless scalar moduli fields survive the projection. The presence of the operator $(1+\Omega)$ in a closed-string one-loop amplitude generates the Klein bottle in addition to the usual toroidal worldsheet. The open-string sector is restricted by the requirement that $X(\sigma)=X(\pi-\sigma)$. In the absence of Chan-Paton factors this kills the states at every odd level, which eliminates the massless photon state as well as the open-string scalar states in the Cartan subalgebra of the group $G$ for generic compactifications. Loops of open string include a factor of $\left(1+(-1)^{N}\right)$, which generates non-orientable surfaces such as the Möbius strip, in addition to the orientable ones.

At enhanced symmetry points there is an ambiguity in how the open-string projection is defined. If the generic projection is applied at these special points there are $\operatorname{dim} G-d$ massless open-string scalars, parameterizing the space $G / U(1)^{d}$. The coupling of $n$ of these coset-space open-string scalar states to a closed string is given by,

$$
\left\langle\text { phys }\left|K^{a_{1}}\left(\sigma_{1}\right) e^{i k_{1} \cdot X\left(\sigma_{1}\right)} \cdots K^{a_{n}}\left(\sigma_{n}\right) e^{i k_{n} \cdot X\left(\sigma_{n}\right)}\right| B\right\rangle
$$

where both the bra and ket are states on which $\Omega=1$. Since $(1+\Omega) K^{a}(1+\Omega)=0$ the coupling vanishes unless $n$ is even so there is no coupling between a single open-string scalar and a single closed string. Therefore, pion states do not mix with closed-string states and the $G$ gauge bosons remain massless as do the $G / U(1)^{d}$ open-string scalars.

Alternatively, at the enhanced symmetry points the non-orientability projection in the open-string sector is naturally generalized to $\left(1+(-1)^{N+p^{2} / 2}\right)$, where $p^{I}$ lies in the (even) root lattice of $G$. In the fermionic formulation this corresponds to treating all the fermions equivalently (whereas the projection of the previous paragraph distinguishes pairs of fermions that form the generators of the Cartan subalgebra). This projection is equivalent to allowing the center of mass, $x$, to wind around the around the compact dimensions only an even number of times. It eliminates all of the massless open-string scalar states. In this case the only massless staes in the theory are in the closed-string sector, namely, the graviton, $\operatorname{dim}(G)$ massless vectors, $\operatorname{dim}(G)$ massless scalars and the dilaton.

The considerations of this paper can be generalized to include non-trivial ChanPaton symmetry factors. Interesting work on the compactification of anomaly-free open superstrings (34 and references cited therein) shows a rich breaking of the Chan-Paton symmetry that is dependent on the background fields of the bulk theory. These theories are naturally non-orientable. 
Acknowledgments

We thank the CERN TH Division for its hospitality and M.Gutperle is grateful for financial support from the EPSRC and a Pannett research studentship from Churchill College, Cambridge.

\section{References}

[1] J.L.Cardy, Conformal invariance and surface critical behaviour, Nucl. Phys. B240 (1984) 514; Effect of boundary conditions on the operator content of two-dimensional conformally invariant theories,Nucl. Phys. B275 (1986) 200; Boundary conditions, fusion rules and the Verlinde formula, Nucl. Phys. B324 (1989) 581.

[2] C.G. Callan and S.R. Das, Boundary conditions on the monopole Dirac equation, Phys. Rev. Lett. 51 (1983) 1155.

[3] I. Affleck and J. Sagi, Monopole catalyzed baryon decay: a boundary conformal field theory approach, hep-th/9311056, Nucl. Phys. B417 (1994) 374.

[4] A.W.W. Ludwig and I. Affleck, Exact conformal field theory results on the multichannel Kondo effect: asymptotic three-dimensional space and time dependent multipoint and many particle Green's functions, Nucl. Phys. B428 (1994) 545-611.

[5] E.Wong and I.Affleck, Tunneling in quantum wires: a boundary conformal field theory approach, Nucl. Phys. B417 (1994) 403.

[6] A.O. Caldeira and A.J. Legget, Path integral approach to quantum brownian motion, Physica 121A (1983) 587; Influence of dissipation on quantum tunnelling in macroscopic systems, Phys. Rev. Lett. 46 (1981) 211; Quantum tunnelling in a dissipative system, Ann of Phys. 149 (1983) 374.

[7] C.G.Callan and L.Thorlacius, Open string theory as dissipative quantum mechanics, Nucl. Phys.B329 (1990) 117.

[8] P. Fendley, A.W.W. Ludwig and H. Saleur, Exact Conductance through Point Contacts in the $\nu=1 / 3$ Fractional Quantum Hall Effect, USC-94-12, PUPT-94-1491, cond-mat/9408068.

[9] C.G.Callan and I.R.Klebanov, Exact $c=1$ boundary conformal field theories, Phys. Rev. Lett. 72 (1994) 1968.

[10] C. G. Callan, I.R. Klebanov, A.W.W. Ludwig andf J.M. Maldacena Exact solution of a boundary conformal field theory, Nucl. Phys. B422 (1994) 17. 
[11] A. Abouelsaood, C.G. Callan, C.R. Nappi and S.A. Yost, Open strings in background gauge fields, Nucl. Phys. B278 (1986) 550.

[12] C.G. Callan, I.R. Klebanov, J.M. Maldacena and A. Yegulalp, Magnetic fields and fractional statistics in boundary conformal field theory, hep-th/9503014 Nucl.Phys. B443 (1995) 444.

[13] A.Yegulalp, New boundary conformal field theories indexed by the simply laced Lie algebras, hep-th/9504104.

[14] J. Polchinski, L. Thorlacius, Free fermion representation of a boundary conformal field theory, hep-th/9404008, Phys. Rev. D50 (1994) 622.

[15] C.G. Callan, C. Lovelace, C.R. Nappi and S.A. Yost, loop corrections to superstring equations of motion, Nucl. Phys. B308 (1988) 221.

[16] D.E. Freed, Reparametrization invariance in some nonlocal 1-D field theories, hepth/9309116, Nucl. Phys.B424 (1994) 628.

[17] C.G. Callan, A.G. Felce and D.E. Freed Critical theories of the dissipative Hofstadter model, hep-th/9202085, Nucl. Phys.B392 (1993) 551.

[18] E. Witten, On background independent open string field theory, hep-th/9208027, Phys.Rev. D46 (1992) 5467.

[19] Keke Li and E. Witten, Role of short distance behavior in off-shell open string field theory, hep-th/9303067, Phys.Rev. D48 (1993) 853.

[20] S.L. Shatashvili, On the problems with background independence in string theory, hep-th/9311177; Comment on the background independence of open string theory, hep-th/9303143, Phys. Lett. B311 (1993) 83.

[21] E. Cremmer and J. Scherk, Spontaneous dynamical symmetry breaking of gauge symmetry in dual models, Nucl. Phys. B72 (1974) 117.

[22] J.A. Shapiro and C.B. Thorn, BRST invariant transitions between closed and open strings, Phys. Rev. D36 (1987) 432.

[23] M.B. Green and P. Wai, The insertion of boundaries in world-sheets, Nucl. Phys. B431 (1993) 131.

[24] N.Ishibashi, The boundary and cross-cap states in conformal field theories, Modern Physics Letters A4 (1989) 251. 
[25] K.S. Narain, M.H. Sarmadi and E. Witten, A note on toroidal compactification of the heterotic string, Nucl. Phys. B279 (1987) 369.

[26] A. Giveon, M. Porrati and E. Rabinovici, Target space duality in string theory, hepth/9401139 Phys. Rep. 244 (1994) 77.

[27] C.G. Callan, C. Lovelace, C.R. Nappi and S.A. Yost, Adding holes and cross-caps to the superstring, Nucl. Phys. B293 (1987) 83.

[28] J. Polchinski and Y. Cai, Consistency of open superstring theories, Nucl. Phys. B296 (1988) 91.

[29] S.Elitzur, E.Gross, E.Rabinovici and N.Seiberg, Aspects of bosonization in string theory, Nucl. Phys. B283 (1987) 413.

[30] W. Fischler and L. Susskind, Dilaton tadpoles, string condensates and scale invariance, Phys. Lett. 171B (1986) 383; Dilaton tadpoles, string condensates and scale invariance. II, Phys. Lett. 173B (1986) 262.

[31] E.S. Fradkin and A.A. Tseytlin, Quantum string theory effective action, Nucl. Phys. B261 ( 1985) 1.

[32] C.G. Callan, D. Friedan, E.J. Martinec and M.J. Perry, Strings in background fields, Nucl. Phys. B262 (1985) 593.

[33] A. Sen, The heterotic string in arbitrary background field, Phys. Rev. D32 (1985) 2102; Equations of motion for the heterotic string from the conformal invariance of the sigma model, Phys. Rev. Lett. 55 (1985) 1846.

[34] G. Pradisi and A. Sagnotti, New developments in open-string theories, hepth/9211084, talk given at 10th Italian Conference on General Relativity and Gravitational Physics. Bardonecchia, Italy, Sep 1992. 